\title{
SPECIAL VINBERG CONES
}

\section{V. ALEKSEEVSKY}

Institute for

Information Transmission Problems

B. Karetnuj per. 19

Moscow, 127051, Russia

and

University of Hradec Králové

Faculty of Science

Rokitanského 62

50003 Hradec Králové

Czech Republic

dalekseevsky@iitp.ru

\section{CORTÉS}

\author{
Department of Mathematics \\ Universität Hamburg \\ Bundesstraße 55 \\ D-20146 Hamburg, Germany
}

vicente.cortes@uni-hamburg.de

To the blessed memory of E. B. Vinberg

\begin{abstract}
The paper is devoted to the generalization of the Vinberg theory of homogeneous convex cones. Such a cone is described as the set of "positive definite matrices" in the Vinberg commutative algebra $\mathcal{H}_{n}$ of Hermitian T-matrices. These algebras are a generalization of Euclidean Jordan algebras and consist of $n \times n$ matrices $A=\left(a_{i j}\right)$, where $a_{i i} \in \mathbb{R}$, the entry $a_{i j}$ for $i<j$ belongs to some Euclidean vector space $\left(V_{i j}, g\right)$ and $a_{j i}=$ $a_{i j}^{*}=g\left(a_{i j}, \cdot\right) \in V_{i j}^{*}$ belongs to the dual space $V_{i j}^{*}$. The multiplication of T-Hermitian matrices is defined by a system of "isometric" bilinear maps $V_{i j} \times V_{j k} \rightarrow V_{i j}, i<j<k$, such that $\left|a_{i j} \cdot a_{j k}\right|=\left|a_{i j}\right| \cdot\left|a_{j k}\right|, a_{l m} \in V_{l m}$. For $n=2$, the Hermitian T-algebra $\mathcal{H}_{2}=$ $\mathcal{H}_{2}(V)$ is determined by a Euclidean vector space $V$ and is isomorphic to a Euclidean Jordan algebra called the spin factor algebra and the associated homogeneous convex cone is the Lorentz cone of timelike future directed vectors in the Minkowski vector space $\mathbb{R}^{1,1} \oplus$ $V$. A special Vinberg Hermitian T-algebra is a rank 3 matrix algebra $\mathcal{H}_{3}(V, S)$ associated to a Clifford $\mathrm{Cl}(V)$-module $S$ together with an "admissible" Euclidean metric $g_{S}$.

We generalize the construction of rank 2 Vinberg algebras $\mathcal{H}_{2}(V)$ and special Vinberg algebras $\mathcal{H}_{3}(V, S)$ to the pseudo-Euclidean case, when $V$ is a pseudo-Euclidean vector space and $S=S_{0} \oplus S_{1}$ is a $\mathbb{Z}_{2}$-graded Clifford $\mathrm{Cl}(V)$-module with an admissible pseudoEuclidean metric. The associated cone $\mathcal{V}$ is a homogeneous, but not convex cone in $\mathcal{H}_{m}, m=2,3$. We calculate the characteristic function of Koszul-Vinberg for this cone and write down the associated cubic polynomial. We extend Baez' quantum-mechanical interpretation of the Vinberg cone $\mathcal{V}_{2} \subset \mathcal{H}_{2}(V)$ to the special rank 3 case.
\end{abstract}

DOI: $10.1007 / \mathrm{s} 00031-021-09649-\mathrm{w}$

Received September 25, 2020. Accepted January 28, 2021.

Published online April 6, 2021.

Corresponding Author: V. Cortés, e-mail: vicente.cortes@uni-hamburg.de

MSC classification: 53C30, 53C26. Keywords: Vinberg algebras, homogeneous cones, special geometry, projective special real manifolds. 


\section{Introduction}

Sixty years ago E. B. Vinberg developed a theory of homogeneous convex cones [30], [31]. He proved that any selfdual homogeneous convex cone is the cone of positive elements $a \in J$ of a Euclidean Jordan algebra $J$. An invertible element $a$ is called positive if it admits a square root $b$, such that $a=b^{2}$.

We recall that Jordan algebras were defined by P. Jordan in 1933 in a paper [22] devoted to the axiomatization of quantum mechanics as a notion which formalized the notion of an algebra of observables. A year later, Euclidean (or formally real) Jordan algebras were classified by the great physicists P. Jordan, J. von Neumann and E. Wigner [23] . They proved that any such algebra is a direct sum of simple algebras. All simple Euclidean Jordan algebras are exhausted by the spin factor algebras $J_{2}(\widehat{V})$ (associated with Euclidean spaces $V$, cf. Section 3.1) and the algebras of rank $n$ Hermitian matrices $\mathcal{H}_{n}(\mathbb{K})$ over division algebras $\mathbb{K}=\mathbb{R}, \mathbb{C}, \mathbb{H}$ or $\mathbb{O}$ (for $n=3$ ). Later Vinberg reduced the classification of homogeneous convex cones to the description of "compact rank $n$ left symmetric normal algebras" (clans). He developed a theory of clans and showed that they can be described in the framework of rank $n$ matrix T-algebras $\mathcal{M}_{n}$ (Vinberg T-algebras). He proved that any homogeneous convex cone is the cone $\mathcal{V}_{n} \subset \mathcal{H}_{n}$ of positively defined matrices in the commutative algebra $\mathcal{H}_{n} \subset \mathcal{M}_{n}$ of Hermitian matrices with the Jordan multiplication $X \circ Y=\frac{1}{2}(X Y+Y X)$. The T-algebras $\mathcal{H}_{n}$ of Hermitian matrices constitute a natural generalization of Euclidean Jordan algebras. In particular, the rank 2 Hermitian T-algebras are isomorphic to the (Euclidean) "spinor factor" Jordan algebras and the associated cone is the Lorentz cone.

This theory has many applications to different parts of physics (quantum physics [6], [7], supergravity [20], [19], [29], quantum field theory and renormalization $[26],[12])$, special Kähler and quaternionic Kähler geometry [1], [3], [13], [14], harmonic analysis [16], information geometry [4], [8], Souriau thermodynamics on Lie groups [8], statistics [4], [17], convex optimization [11], combinatorics [12], numerical integration of differential equations [21], etc.

A Vinberg rank $n$ matrix T-algebra $\mathcal{M}_{n}$ consists of $n \times n$ matrices $X=\left(x_{i j}\right)$ where $x_{i i} \in \mathbb{R}$ and off-diagonal elements $x_{i j}$ belong to different Euclidean vector spaces $V_{i j}$ such that the space $V_{j i}=V_{i j}^{*}$ is dual to $V_{i j}$. To define multiplication of matrices, Vinberg postulated the existence of some "isometric" bilinear maps

$$
V_{i j} \otimes V_{j k} \rightarrow V_{i k}, x_{i j} \otimes y_{j k} \mapsto x_{i j} \cdot y_{j k}
$$

such that

$$
\left|x_{i j} \cdot y_{j k}\right|=\left|x_{i j}\right| \cdot\left|y_{j k}\right|
$$

and some additional axioms hold.

The problem of classification of isometric maps $\varphi: U \otimes V \rightarrow W$ between Euclidean vector spaces is an important, but very complicated, open problem. It was solved only in the case of spaces of the same dimension by A. Hurwitz (in his theory of division algebras, 1898) and in the case when $\operatorname{dim} V=\operatorname{dim} W$ by M. F. Atiyah, R. Bott and A. S. Shapiro (theory of Clifford modules, 1964). The first case corresponds to Euclidean Jordan algebras and selfdual homogeneous convex cones (in particular, rank two Vinberg cones). The second case corresponds to rank 
three special Vinberg T-algebras and special Vinberg cones. Practically, only these two classes of Vinberg cones are used in physics and other applications.

The main aim of our paper is to generalize the construction of Vinberg cones of rank 2 and special Vinberg cones of rank 3 to the indefinite setting. More precisely, a rank 2 Vinberg T-algebra of dimension $2 n+2$ is defined by a Euclidean $n$-dimensional vector space $V$ and the associated Hermitian Vinberg algebra is identified with the $n+2$ dimensional Minkowski vector space which has the structure of a spin factor Jordan algebra. Similarly, a rank 3 special Vinberg algebra $\mathcal{M}_{3}(V, S)$ is defined by a Euclidean vector space $\left(V, g_{V}\right)$ and a Clifford $\mathrm{Cl}(V)$ module $S$ with admissible Euclidean metric $g_{S}$. Admissibility means here that the operator $\mu_{v}: s \mapsto v \cdot s$ of Clifford multiplication by a unit vector is orthogonal.

We generalize these constructions to the indefinite case, where $V$ is a pseudoEuclidean vector space and $S=S_{0} \oplus S_{1}$ is a $\mathbb{Z}_{2}$-graded Clifford $\mathrm{Cl}(V)$-module with admissible pseudo-Euclidean metric $g_{S}$. In particular, we define corresponding algebras $\mathcal{M}_{2}(V)$ and $\mathcal{M}_{3}(V, S)$ and show that they satisfy Vinberg's axioms of a T-algebra with the exception of the positivity axiom, see Proposition 3 . With such indefinite rank 2 or special rank 3 algebras $\mathcal{M}_{2}(V)$ or $\mathcal{M}_{3}(V, S)$ we associate an open homogeneous, but not convex cone $\mathcal{V}$ in the associated commutative algebra $\mathcal{H}_{k}, k=2,3$, of Hermitian matrices, see Theorem 1 and Theorem 2. As in the positive definite case, the solvable group of upper triangular matrices acts simply transitively in the cone $\mathcal{V}$. We describe the system of inequalities, which defines $\nu$ and calculate the characteristic Koszul-Vinberg function $\varphi$ (the density of the invariant measure) which plays an important role in applications, see Theorem 3

For example, it defines a canonical Hessian Riemannian metric (the Hessian of $\log \varphi$ ). In information geometry, Vinberg cones are identified with an important class of manifolds of probability distributions (so-called "exponential family") and the canonical metric with the Fisher metric. In Souriau thermodynamics, the Legendre transform of $\log \varphi$ is interpreted as an entropy [8]. In convex optimization, the function $\varphi$ is used as a barrier function, which allows us to apply the Newton methods for the determination of the minimum of the given function.

We calculate also the cubic polynomial $h$, associated with the characteristic function. The characteristic hypersurface $\{h=1\} \subset \mathcal{V}$ defines a projective special real manifold, which is the Riemannian scalar manifold of a supergravity theory in five Lorentzian space-time dimensions (when the metrics $g_{V}, g_{S}$ are Euclidean). It is known that such manifolds give rise to projective special Kähler and quaternionic Kähler manifolds by dimensional reduction of the underlying supersymmetric field theories [29]. In fact, the reduction of the space-time dimension of the theory (from 5 to 4 and further down to 3 ) implies an increase in the number scalar fields, and the geometry of the corresponding higher dimensional scalar field space is eventually special Kähler or quaternionic Kähler by supersymmetry. In particular, homogeneous cones with cubic potential $h$ are related to homogeneous special Kähler and quaternionic Kähler manifolds [29]. More generally, examples of complete quaternionic Kähler manifolds with small cohomogeneity can be obtained in this way, see [15] for the first such examples of cohomogeneity one.

The cubic polynomial is not only the exponential of the Hesse potential of the projective special real manifold but is also related to various potentials governing 
the corresponding special Kähler geometry and to the entropy of black holes, see, e.g., [10], [27], [25].

John Baez [6] gave a nice quantum mechanical interpretation of the rank 2 Vinberg cones in the case when $V=\mathbb{K}$ is a division algebra. We extend this interpretation to all rank 2 and special rank 3 Vinberg cones by showing that the elements of the Vinberg cones can be considered as symmetric endomorphisms satisfying a certain positivity condition, see Proposition 4 and Theorem 3, part i).

The section of a Vinberg cone by an affine hyperplane is a domain, and not necessarily homogeneous. However, Vinberg proved that any homogeneous convex domain can be realized as a codimension 1 section of a classical (i.e., convex) Vinberg cone. A codimension 1 section through the origin, defines a cone. In the last section, we consider the problem of the description of such sections which are homogeneous cones or have small cohomogeneity.

In the definite case, the homogeneous examples associated with Vinberg cones or codimension one sections thereof include the homogeneous cones over the homogeneous projective special real manifolds classified in [29], which are related to the homogeneous quaternionic Kähler manifolds described in [1], [13] as solvable Lie groups with left-invariant quaternionic Kähler structure, see Remarks 5-7 and Example 1. The inhomogeneous examples include complete convex cones of cohomogeneity one, such as the cones over the complete projective special real manifolds of cohomogeneity one found in [15], see Example 1.

In the indefinite case, we obtain further examples of cones of small cohomogeneity, which are no longer convex and correspond to indefinite quaternionic Kähler manifolds of small cohomogeneity, including homogeneous examples, cf. Theorem 2, Proposition 11, Proposition 8, Remark 6 and Remark 8.

Acknowledgements. This work was partially supported by grant no. 18-00496S of the Czech Science Foundation and by the German Science Foundation (DFG) under Germany's Excellence Strategy-EXC 2121 "Quantum Universe"-390833306.

\section{T-algebras of rank 3 associated with metric Clifford modules}

Let $\left(V, g_{V}\right)$ be a pseudo-Euclidean vector space and $S=S_{0}+S_{1}$ a $\mathbb{Z}_{2}$-graded module over the Clifford algebra $\mathrm{Cl}(V)$ endowed with an admissible pseudo-Euclidean scalar product $g_{S}$. Recall [2] that a pseudo-Euclidean metric $g_{S}$ in $S$ is called admissible if it has a type $\tau \in\{ \pm 1\}$, that is, if the Clifford multiplication

$$
\mu_{v}: S \rightarrow S, \quad s \mapsto \mu_{v} s
$$

is either $g_{S}$-symmetric for all $v \in V(\tau=1)$ or $g_{S}$-skew-symmetric $(\tau=-1)$. We will also assume that $g_{S}$ is even, that is $g_{S}\left(S_{0}, S_{1}\right)=0$.

We will follow the convention in which the Clifford relation is

$$
\mu_{v}^{2}=-g_{V}(v, v) \operatorname{Id}_{S}, \quad v \in V .
$$

We call $\left(S, g_{S}\right)$ a $\mathbb{Z}_{2}$-graded metric Clifford module. The description of such pairs $\left(S, g_{S}\right)$ is given in [2]. 
For all $i, j \in\{1,2,3\}$ we define vector spaces $\mathcal{A}_{i j}$ as follows:

$$
\mathcal{A}_{i i}=\mathbb{R}, \mathcal{A}_{12}=V, \mathcal{A}_{21}=V^{*}, \mathcal{A}_{23}=S_{0}, \mathcal{A}_{32}=S_{0}^{*}, \mathcal{A}_{13}=S_{1}, \mathcal{A}_{31}=S_{1}^{*} .
$$

We denote by

$$
\begin{aligned}
& *: V \rightarrow V^{*}, v \mapsto v^{*}=g_{V} v:=g_{V}(v, \cdot), \\
& *: S_{i} \rightarrow S_{i}^{*}, s \mapsto s^{*}=g_{S} s \in\left(S_{i}^{\perp}\right)^{\mathrm{ann}} \cong S_{i}^{*}
\end{aligned}
$$

the natural isomorphisms, where $\left(S_{i}^{\perp}\right)^{\text {ann }} \subset S^{*}$ is canonically identified with $S_{i}^{*}$. Definining $*: \mathbb{R} \rightarrow \mathbb{R}$ as the identity, this defines a system of isomorphisms $*: \mathcal{A}_{i j} \rightarrow \mathcal{A}_{j i}$ such that $*^{2}=\mathrm{Id}$. We will refer to $*$ as the conjugation (map) and to $a^{*}$ as the conjugate of $a$.

Proposition 1. Define bilinear maps

$$
\mathcal{A}_{i j} \times \mathcal{A}_{j k} \rightarrow \mathcal{A}_{i k}
$$

as follows.

(1) If $i=j$ or $j=k$, the product (2) is defined by scalar multiplication and if $i=k \neq j$, the product is defined by the natural pairing.

(2) The product

$$
\mathcal{A}_{12} \times \mathcal{A}_{23}=V \times S_{0} \rightarrow \mathcal{A}_{13}=S_{1},(v, s) \mapsto v \cdot s:=\mu_{v} s
$$

is defined by Clifford multiplication.

(3) The product

$$
\mathcal{A}_{23} \times \mathcal{A}_{31}=S_{0} \times S_{1}^{*} \rightarrow \mathcal{A}_{21}=V^{*}
$$

is defined by

$$
\left(s_{0} \cdot s_{1}^{*}\right)(v)=\left(s_{0} \cdot s_{1}^{*}\right) \cdot v=s_{1}^{*} \cdot\left(v \cdot s_{0}\right)=s_{1}^{*}\left(v \cdot s_{0}\right),
$$

and

$$
\mathcal{A}_{31} \times \mathcal{A}_{12}=S_{1}^{*} \times V \rightarrow \mathcal{A}_{32}=S_{0}^{*}
$$

by

$$
\left(s_{1}^{*} \cdot v\right)\left(s_{0}\right)=\left(s_{1}^{*} \cdot v\right) \cdot s_{0}=s_{1}^{*} \cdot\left(v \cdot s_{0}\right)=s_{1}^{*}\left(v \cdot s_{0}\right),
$$

where $v \in V, s_{0} \in S_{0}$ and $s_{1}^{*} \in S_{1}^{*}$.

(4) The remaining products are defined by:

$$
s_{0}^{*} \cdot v^{*}:=\left(v \cdot s_{0}\right)^{*} \in S_{1}^{*}, s_{1} \cdot s_{0}^{*}:=\left(s_{0} \cdot s_{1}^{*}\right)^{*} \in V, v^{*} \cdot s_{1}:=\left(s_{1}^{*} \cdot v\right)^{*} \in S_{0},
$$

where $v \in V, s_{0} \in S_{0}, s_{1} \in S_{1}$ and $v^{*} \in V^{*}, s_{0}^{*} \in S_{0}^{*}, s_{1}^{*} \in S_{1}^{*}$ are their conjugates.

Then

$$
\left(a_{i j} a_{j k}\right)^{*}=a_{j k}^{*} a_{i j}^{*}
$$

for all $a_{i j} \in \mathcal{A}_{i j}, a_{j k} \in \mathcal{A}_{j k}$. 
Proof. When two of the indices $i, j, k$ coincide, the claim is trivially satisfied. Otherwise $(i, j, k)$ is a permutation of $(1,2,3)$. For an even permutation, (6) follows directly from (5). The case of an odd permutation follows by applying the conjugation map to $(6)$.

For future use we state the following lemma.

Lemma 1. For all $v \in V, s_{i} \in S_{i}$ we have:

$$
g_{S}\left(s_{0}, v^{*} \cdot s_{1}\right)=g_{S}\left(v \cdot s_{0}, s_{1}\right)
$$

and, hence, $v^{*} \cdot s_{1}=\tau \mu_{v} s_{1}$, where $\tau$ is the type of $g_{S}$.

Proof. We put $t_{0}:=\left(s_{1}^{*} \cdot v\right)^{*} \in S_{0}$. Then

$$
g_{S}\left(s_{0}, v^{*} \cdot s_{1}\right)=g_{S}\left(s_{0}, t_{0}\right)=t_{0}^{*} \cdot s_{0}=\left(s_{1}^{*} \cdot v\right) \cdot s_{0} \stackrel{(4)}{=} g_{S}\left(s_{1}, v \cdot s_{0}\right) .
$$

We denote by

$$
\mathcal{A}=\bigoplus_{i, j=1}^{3} \mathcal{A}_{i j}=\mathbb{R}^{3} \oplus W \oplus W^{*}, \quad W:=V \oplus S,
$$

the space of matrices $A=\left(a_{i j}\right)_{i, j=1,2,3}$ with $a_{i j} \in \mathcal{A}_{i j}$.

Definition 1. For any $A \in \mathcal{A}$ we define the conjugate matrix $A^{*} \in \mathcal{A}$ by its matrix elements $\left(A^{*}\right)_{i j}=a_{j i}^{*}$. We can express this relation as $A^{*}=\left(a_{j i}^{*}\right)_{i j}$. $\mathrm{A}$ matrix $A \in \mathcal{A}$ is called Hermitian if $A^{*}=A$.

Proposition 2. The bilinear maps (2) defined above induce a unital product on the space $\mathcal{A}$ such that $(A B)^{*}=B^{*} A^{*}$ for all $A, B \in \mathcal{A}$.

Proof. The product $C=A B=\left(c_{i j}\right)$ is defined by $c_{i j}=\sum a_{i k} b_{k j}$. So $\left(C^{*}\right)_{j i}=$ $c_{i j}^{*}=\sum b_{k j}^{*} a_{i k}^{*}=\left(B^{*} A^{*}\right)_{j i}$. This proves that $C^{*}=B^{*} A^{*}$. The unit matrix $\mathbf{1}=$ $\left(\delta_{i j}\right)$ is a neutral element.

Proposition 3. The algebra $\mathcal{A}=\mathcal{A}(V, S)$ associated with the Clifford module $\left(S, g_{S}\right)$ with metric satisfies Vinberg's axioms of a T-algebra with the exception of the positivity axiom, which holds if and only if the metrics $g_{V}$ and $g_{S}$ are positive definite, and the axiom 4, which holds if and only if the type of $g_{S}$ is $\tau=-1$. More precisely, $\mathcal{A}$ is a generalized T-algebra of rank 3, as defined after the proof of this proposition.

Proof. We recall and check the five axioms [30]:

1) $\mathcal{A}_{i i} \cong \mathbb{R}$ holds.

2) $a_{i j} \cdot a_{i j}^{*}>0$ for all $a_{i j} \in \mathcal{A}_{i j} \backslash\{0\}$ (positivity axiom) holds if and only if the metrics are positive definite. This is clear, since $v \cdot v^{*}=v^{*} \cdot v=g_{V}(v, v)$ for all $v \in \mathcal{A}_{12}=V$ and $s \cdot s^{*}=s^{*} \cdot s=g_{S}(s, s)$ for all $s \in \mathcal{A}_{23} \cup \mathcal{A}_{13}=S_{0} \cup S_{1}$.

3) $a_{i j} \cdot\left(b_{j k} \cdot c_{k \ell}\right)=\left(a_{i j} \cdot b_{j k}\right) \cdot c_{k \ell}$ for all $a_{i j} \in \mathcal{A}_{i j}, b_{j k} \in \mathcal{A}_{j k}, c_{k \ell} \in \mathcal{A}_{k \ell}$, if $i \neq k$ and $j \neq \ell$ (associativity axiom). It is clear that the axiom holds if $i=j$ or $j=k$ or $k=\ell$. So we are left with the six permutations $(i, j, k)$ of $(1,2,3)$ and $\ell=i$. We check these cases. 
Case $(i, j, k)=(1,2,3)$ :

$$
\left(v \cdot s_{0}\right) \cdot s_{1}^{*}=s_{1}^{*} \cdot\left(v \cdot s_{0}\right) \stackrel{(3)}{=}\left(s_{0} \cdot s_{1}^{*}\right) \cdot v=v \cdot\left(s_{0} \cdot s_{1}^{*}\right), \quad v \in V, s_{i} \in S_{i} .
$$

Case $(i, j, k)=(2,3,1)$. Using the previous equation we calculate

$$
\left(s_{0} \cdot s_{1}^{*}\right) \cdot v=s_{1}^{*} \cdot\left(v \cdot s_{0}\right) \stackrel{(4)}{=}\left(s_{1}^{*} \cdot v\right) \cdot s_{0}=s_{0} \cdot\left(s_{1}^{*} \cdot v\right) .
$$

Case $(i, j, k)=(3,1,2)$ is $(4)$ and the remaining cases are obtained by conjugation.

4) If $j$ lies between $i$ and $k$, then $a_{i j}^{*} \cdot\left(a_{i j} \cdot b_{j k}\right)=\left(a_{i j}^{*} \cdot a_{i j}\right) \cdot b_{j k}$ for all $a_{i j} \in \mathcal{A}_{i j}$, $b_{j k} \in \mathcal{A}_{j k}$. The axiom is trivially satisfied if some of the indices coincide. So we are left with the case $(i, j, k)=(1,2,3)$, which amounts to the equation

$$
v^{*} \cdot(v \cdot s)=\left(v^{*} \cdot v\right) \cdot s=g_{V}(v, v) s, \quad v \in V, s \in S_{0} .
$$

The left-hand side coincides with $\tau\left(\mu_{v}\right)^{2} s$, in virtue of Lemma 1. So the axiom holds if and only if $\tau=-1$, cf. (1).

5) There exist positive numbers $n_{i}$ such that $n_{i} a_{i j} \cdot b_{i j}^{*}=n_{j} b_{i j} \cdot a_{i j}^{*}$ for all $a_{i j}, b_{i j} \in \mathcal{A}_{i j}$. This axiom holds with $n_{i}=1$ due to the symmetry of the metrics.

Note that we could restate Proposition 3 by saying that every Clifford module with metric defines a generalized T-algebra of rank 3 , if we define such an algebra by the axioms 1$), 3), 5$ ), replacing 2 ) by the condition that $a_{i j} \cdot a_{i j}^{*}$ is a nondegenerate quadratic form on $\mathcal{A}_{i j}$ and replacing $a_{i j}^{*} \cdot\left(a_{i j} \cdot b_{j k}\right)=\left(a_{i j}^{*} \cdot a_{i j}\right) \cdot b_{j k}$ by $a_{i j}^{*} \cdot\left(a_{i j} \cdot b_{j k}\right)=-\tau\left(a_{i j}^{*} \cdot a_{i j}\right) \cdot b_{j k}$ in axiom 4 .

Definition 2. A generalized T-algebra $\mathcal{A}$ of rank 3 for which $\tau=-1$ (i.e., for which the T-algebra axiom 4 holds) will be called a (possibly indefinite) $T$-algebra of rank 3. The $T$-algebra $\mathcal{A}$ of rank 3 will be called a special T-algebra if either $\operatorname{dim} \mathcal{A}_{13}=\operatorname{dim} \mathcal{A}_{23}$ or if $\operatorname{dim} \mathcal{A}_{12}=0$.

With this definition the special T-algebras are precisely the algebras $\mathcal{A}(V, S)$ defined above.

\subsection{The solvable group $G(W)$ and its action on the space of Hermitian matrices $\operatorname{Herm}(\boldsymbol{W})$}

The set $G=G(W)$ of upper triangular matrices $A \in \mathcal{A}$ of the form

$$
A=\left(\begin{array}{ccc}
a_{11} & a_{12} & a_{13} \\
0 & a_{22} & a_{23} \\
0 & 0 & a_{33}
\end{array}\right)
$$

with positive diagonal elements $a_{i i}>0$ is a solvable connected and simply connected Lie group.

We denote by $\mathfrak{g}=\mathfrak{g}(W)$ its Lie algebra. It consists of matrices of the form (7) with arbitrary diagonal elements $a_{i i} \in \mathbb{R}$. 
Let us denote by $\operatorname{Herm}(W) \subset \mathcal{A}$ the space of Hermitian matrices

$$
X=\left(\begin{array}{ccc}
X_{11} & X_{12} & X_{13} \\
X_{12}^{*} & X_{22} & X_{23} \\
X_{13}^{*} & X_{23}^{*} & X_{33}
\end{array}\right)=\left(\begin{array}{ccc}
x_{1} & X_{3} & X_{2} \\
X_{3}^{*} & x_{2} & X_{1} \\
X_{2}^{*} & X_{1}^{*} & x_{3}
\end{array}\right),
$$

where $X_{3}=X_{12} \in V, X_{1}=X_{23} \in S_{0}, X_{2}=X_{13} \in S_{1}$ and $x_{i}=X_{i i} \in \mathbb{R}$, and define a map

$$
\begin{aligned}
\mathfrak{g}(W) \times \operatorname{Herm}(W) & \rightarrow \operatorname{Herm}(W), \\
(A, X) & \mapsto T_{A} X:=A X+X A^{*}
\end{aligned}
$$

Remark 1. Note that with $A_{t}:=\exp (t A) \in G(W), A \in \mathfrak{g}(W)$ we have

$$
T_{A} X=\left.\frac{d}{d t}\right|_{t=0}\left(\left(A_{t} X\right) A_{t}^{*}+A_{t}\left(X A_{t}^{*}\right)\right) .
$$

Remark 2. Note that the expression differentiated on the right-hand side of (9) is Hermitian in virtue of Proposition 2. It cannot be simplified, since $\mathcal{A}$ is not associative. The obstruction to $(A X) A^{*}=A\left(X A^{*}\right)$ for $A \in G(W)$ is precisely that $\left(v \cdot s_{0}\right) \cdot \tilde{s}_{0}^{*} \in V$ does in general not coincide with $v \cdot\left(s_{0} \cdot \tilde{s}_{0}^{*}\right)=g_{S}\left(s_{0}, \tilde{s}_{0}\right) v$, $v \in V, s_{0}, \tilde{s}_{0} \in S_{0}$. In fact, from Proposition 1 we obtain for all $u \in V$ :

$$
\begin{aligned}
g_{V}\left(\left(v \cdot s_{0}\right) \cdot \tilde{s}_{0}^{*}, u\right) & =\left(\tilde{s}_{0} \cdot\left(v \cdot s_{0}\right)^{*}\right)(u)=\left(v \cdot s_{0}\right)^{*}\left(u \cdot \tilde{s}_{0}\right) \\
& =g_{S}\left(v \cdot s_{0}, u \cdot \tilde{s}_{0}\right)=\tau g_{S}\left(\mu_{u} \mu_{v} s_{0}, \cdot \tilde{s}_{0}\right) \\
& =-\tau g_{V}(u, v) g_{S}\left(s_{0}, \tilde{s}_{0}\right)+\tau g_{S}\left([u, v] \cdot s_{0}, \tilde{s}_{0}\right) .
\end{aligned}
$$

So under the assumption that $S_{0} \neq 0$, we see that equality $\left(v \cdot s_{0}\right) \cdot \tilde{s}_{0}^{*}=v \cdot\left(s_{0} \cdot \tilde{s}_{0}^{*}\right)$ holds for all $v, s_{0}, \tilde{s}_{0}$ if and only if $\tau=-1$ and $\operatorname{dim} V \leq 1$.

From the above calculation one can easily deduce that if $S_{0} \neq 0$, then there exist elements $A=\mathbf{1}+v+s_{0} \in G(W)$ and $X=\tilde{s}_{0}+\tilde{s}_{0}^{*} \in \operatorname{Herm}(W)$, where $v \in V$ and $s_{0}, \tilde{s}_{0} \in S_{0}$, such that $(A X) A^{*} \neq A\left(X A^{*}\right)$, unless $\tau=-1$ and $\operatorname{dim} V \leq 1$.

Theorem 1. Let $\mathcal{A}$ be a T-algebra of rank 3 associated with a graded $\mathrm{Cl}(V)$ module $S$ and an admissible scalar product $g_{S}$ of type $\tau=-1$. Then the formula (8) defines a representation of the Lie algebra $\mathfrak{g}(W)$ on the space $\operatorname{Herm}(W)$.

Proof (of Theorem 1). The calculation in Remark 2 implies the following lemma, since any admissible bilinear form is $\mathfrak{s p i n}(V)$-invariant.

Lemma 2. Let $\mathcal{A}$ be a generalized T-algebra of rank 3 associated with a graded $\mathrm{Cl}(V)$ module $S$ and an admissible scalar product $g_{S}$ of type $\tau$. Then the following identity holds

$$
\left(v \cdot s_{0}\right) \cdot \tilde{s}_{0}^{*}+\left(v \cdot \tilde{s}_{0}\right) \cdot s_{0}^{*}=-2 \tau v \cdot\left(s_{0} \cdot \tilde{s}_{0}^{*}\right),
$$

for all $v \in V, s_{0}, \tilde{s}_{0} \in S_{0}$.

Using Proposition 1, Proposition 2 and Lemma 2 with $\tau=-1$ one can check that $T_{A} X:=A X+X A^{*}$ defines a representation of $\mathfrak{g}=\operatorname{Lie} G$ on $\operatorname{Herm}(W)$. The lemma is used to check that $\left[T_{A}, T_{B}\right]=T_{[A, B]}$ when $A \in \mathcal{A}_{12}=V, B \in \mathcal{A}_{23}=S_{0}$. The other cases are straightforward. 
Corollary 1. The formula

$$
\begin{aligned}
\exp (t A) \cdot X:= & \exp \left(t T_{A}\right) X=X+T_{A} X+\frac{1}{2} T_{A}^{2} X+\cdots \\
= & X+A X+X A^{*} \\
& +\frac{1}{2}\left(A(A X)+A\left(X A^{*}\right)+(A X) A^{*}+\left(X A^{*}\right) A^{*}\right)+\cdots,
\end{aligned}
$$

where $A \in \mathfrak{g}(W), X \in \operatorname{Herm}(W)$ defines a representation of the group $G(W)$ on $\operatorname{Herm}(W)$.

\subsection{The space of Hermitian matrices $\operatorname{Herm}(W)$ as a space of symmetric endomorphisms of $W$}

We decompose $W=V \oplus S$ as

$$
W=W_{1} \oplus W_{2} \oplus W_{3}, \quad \text { where } \quad W_{1}=S_{1}, W_{2}=S_{0} \quad \text { and } \quad W_{3}=V .
$$

Then

$$
\mathcal{A}=\mathcal{A}(W)=\left\{A=\left(\begin{array}{ccc}
x_{1} & X_{3} & X_{2} \\
Y_{3}^{*} & x_{2} & X_{1} \\
Y_{2}^{*} & Y_{1}^{*} & x_{3}
\end{array}\right) \mid x_{i} \in \mathbb{R}, X_{i}, Y_{i} \in W_{i}\right\} .
$$

The multiplications $\mathcal{A}_{i j} \times \mathcal{A}_{j k}$ include the following maps among the $W_{i}$ and their dual spaces

$$
\begin{array}{ll}
W_{3} \times W_{1} \rightarrow W_{2}, & W_{2} \times W_{1}^{*} \rightarrow W_{3}, \quad W_{1} \times W_{2}^{*} \rightarrow W_{3}^{*}, \\
W_{3}^{*} \times W_{2} \rightarrow W_{1}, & W_{2}^{*} \times W_{3} \rightarrow W_{1}^{*}, \quad W_{1}^{*} \times W_{3}^{*} \rightarrow W_{2}^{*} .
\end{array}
$$

Therefore identifying $W_{i}$ and $W_{i}^{*}$ with the help of the scalar product $g_{W}=g_{V} \oplus g_{S}$ on $W=V \oplus S=\oplus_{i=1} W_{i}$ we obtain multiplications

$$
W_{i} \times W_{j} \rightarrow W_{k}
$$

for all permutations $(i, j, k)$ of $\{1,2,3\}$. Note that one needs to distinguish the Vinberg multiplication $W_{3} \times W_{2}=\mathcal{A}_{12} \times \mathcal{A}_{13}=V \times S_{1} \rightarrow \mathcal{A}_{23}=W_{1}=S_{0}$ from the Clifford multiplication. They are related by

$$
v \cdot s_{1}:=v^{*} \cdot s_{1}=\tau \mu_{v} s_{1}
$$

for all $v \in V, s_{1} \in S_{1}$, whereas $v \cdot s_{0}=\mu_{v} s_{0}$ when $s_{0} \in S_{0}$.

We define a bilinear map

$$
\mathcal{A}(W) \times W \rightarrow W, \quad(A, w) \mapsto A w,
$$

by

$$
A w:=\left(\begin{array}{c}
x_{1} w_{1}+X_{3} \cdot w_{2}+X_{2} \cdot w_{3} \\
Y_{3}^{*} \cdot w_{1}+x_{2} w_{2}+X_{1} \cdot w_{3} \\
Y_{2}^{*} \cdot w_{1}+Y_{1}^{*} \cdot w_{2}+x_{3} w_{3}
\end{array}\right)=\left(\begin{array}{c}
x_{1} w_{1}+X_{3} \cdot w_{2}+X_{2} \cdot w_{3} \\
Y_{3} \cdot w_{1}+x_{2} w_{2}+X_{1} \cdot w_{3} \\
Y_{2} \cdot w_{1}+Y_{1} \cdot w_{2}+x_{3} w_{3}
\end{array}\right)
$$

where $w_{i} \in W_{i}$ and $w=w_{1}+w_{2}+w_{3} \in W$ and $A$ is as in (10). 
Proposition 4. The endomorphism $M_{A}=(w \mapsto A w) \in \operatorname{End}(W)$ associated with an element $A \in \mathcal{A}(W)$ is $g_{W}$-symmetric if and only if $A \in \operatorname{Herm}(W)$, that is if and only if $X_{i}=Y_{i}$ when $A$ is expressed as in (10).

Proof. Since $M_{A}$ is obviously symmetric, when $A$ is diagonal, we can assume that $x_{1}=x_{2}=x_{3}$. In virtue of Lemma 1 , the restriction of $g_{W}(A \cdot, \cdot)$ to $W_{1} \oplus W_{2}=S$ is symmetric if and only $X_{3}=Y_{3}$. Moreover, we can assume that $X_{3}=Y_{3}=0$, since $M_{A}$ is $g_{W}$-symmetric if $X_{1}=Y_{1}=0, X_{2}=Y_{2}=0$ and $X_{3}=Y_{3}$. Next we evaluate $g_{W}(A \cdot, \cdot)$ on $w, w^{\prime} \in W_{2} \oplus W_{3}$ :

$$
\begin{aligned}
g_{W}\left(A w, w^{\prime}\right) & =g_{W}\left(X_{1} \cdot w_{3}, w_{2}^{\prime}\right)+g_{W}\left(Y_{1} \cdot w_{2}, w_{3}^{\prime}\right) \\
& =\left(X_{1}^{*} \cdot w_{3}^{*}\right)\left(w_{2}^{\prime}\right)+\left(Y_{1} \cdot w_{2}^{*}\right)\left(w_{3}^{\prime}\right) \\
& \stackrel{(5),(3)}{=} g_{S}\left(w_{3} \cdot X_{1}, w_{2}^{\prime}\right)+g_{S}\left(w_{3}^{\prime} \cdot Y_{1}, w_{2}\right) \\
& =\tau\left(g_{S}\left(X_{1}, \mu_{w_{3}} \cdot w_{2}^{\prime}\right)+g_{S}\left(Y_{1}, \mu_{w_{3}^{\prime}} \cdot w_{2}\right)\right) .
\end{aligned}
$$

This shows that the restriction of $g_{W}(A \cdot, \cdot)$ to $W_{2} \oplus W_{3}$ is symmetric if and only if $X_{1}=Y_{1}$. Moreover, we see that $M_{A}$ is $g_{W}$-symmetric if $X_{2}=Y_{2}=0, X_{3}=Y_{3}=0$ and $X_{1}=Y_{1}$. Thus we can assume that $X_{1}=Y_{1}=0$. Finally we evaluate $g_{W}(A \cdot, \cdot)$ on $w, w^{\prime} \in W_{1} \oplus W_{3}$ :

$$
\begin{aligned}
g_{W}\left(A w, w^{\prime}\right) & =g_{S}\left(X_{2} \cdot w_{3}, w_{1}^{\prime}\right)+g_{V}\left(Y_{2} \cdot w_{1}, w_{3}^{\prime}\right) \\
& =\left(X_{2}^{*} \cdot w_{3}\right)\left(w_{1}^{\prime}\right)+g_{V}\left(Y_{2} \cdot w_{1}^{*}, w_{3}^{\prime}\right) \\
(4), \underline{(5),(3)} & g_{S}\left(X_{2}, w_{3} \cdot w_{1}^{\prime}\right)+g_{S}\left(Y_{2}, w_{3}^{\prime} \cdot w_{1}\right) .
\end{aligned}
$$

As above, this shows that the restriction of $g_{W}(A \cdot, \cdot)$ to $W_{1} \oplus W_{3}$ is symmetric if and only if $X_{2}=Y_{2}$. Moreover, we see that $M_{A}$ is $g_{W}$-symmetric if and only if $X_{i}=Y_{i}$ for all $i$.

\section{Rank 2 indefinite Vinberg-Jordan algebra and Vinberg cone}

\subsection{Rank 2 indefinite Vinberg-Jordan algebra}

Let $\left(V, g=g_{V}\right)$ be an $n$-dimensional pseudo-Euclidean vector space of signature $(p, q)$. We denote by $*: V \rightarrow V^{*}, v \mapsto v^{*}:=g v=g(v, \cdot)$ the metric isomorphism. The space

$$
\mathcal{M}_{2}=\mathcal{M}_{2}(V)=\left\{X=\left(\begin{array}{ll}
X_{11} & X_{12} \\
X_{21} & X_{22}
\end{array}\right) \mid X_{11}, X_{22} \in \mathbb{R}, X_{12} \in V, X_{21} \in V^{*}\right\}
$$

with the matrix multiplication is a non-associative algebra, called the T-matrix algebra of rank 2 . The determinant $\operatorname{det} X=X_{11} X_{22}-\left\langle X_{21}, X_{12}\right\rangle$ is a non-multiplicative function : $\operatorname{det} X Y \neq \operatorname{det} X \cdot \operatorname{det} Y$ in general. The subspace

$$
W=\mathcal{H}_{2}(V)=\left\{X=\left(\begin{array}{cc}
x_{1} & v \\
v^{*} & x_{2}
\end{array}\right)=\left(\begin{array}{cc}
t+x_{0} & v \\
v^{*} & t-x_{0}
\end{array}\right)\right\} \subset \mathcal{M}_{2}(V)
$$

of Hermitian matrices is closed under the Jordan multiplication $X \circ Y:=\frac{1}{2}(X Y+$ $\left.Y^{*} X^{*}\right)$. The minus determinant is a quadratic form given by 


$$
g(X, X):=-\operatorname{det} X=-x_{1} x_{2}+|v|^{2}=-t^{2}+x_{0}^{2}+|v|^{2}=\frac{1}{2} \operatorname{tr}(\tilde{X} X),
$$

where $|v|^{2}:=g(v, v)$. Here $\tilde{X}=X-\operatorname{tr}(X)$ Id is the trace inversion, defined by Schray [28]. It changes the sign of the time coordinate $t$. We denote by $g(X, Y)$ the associated pseudo-Riemannian metric of signature $(p+1, q+1)$, given by

$$
g(X, Y)=\frac{1}{2} \operatorname{tr}(\tilde{X} Y)=\frac{1}{2} \operatorname{tr}(X \tilde{Y}) .
$$

If $V$ Euclidean $n$-space then $\left(W=\mathcal{H}_{2}(V), g\right)$ is the Minkowski space of dimension $n+2$.

Proposition 5. The algebra $\left(\mathcal{H}_{2}(V), \circ\right)$ is a Jordan algebra isomorphic to the pseudo-Euclidean spin factor algebra

$$
J_{2}(\widehat{V})=\mathbb{R} 1+\widehat{V}
$$

associated with the pseudo-Euclidean vector space $\widehat{V}:=\mathbb{R} \stackrel{\perp}{\oplus} V$ of signature $(p+$ $1, q)$, with unit 1 and multiplication

$$
(t, \widehat{u}) \circ(s, \widehat{v})=(t s+g(\widehat{u}, \widehat{v}), t \widehat{v}+s \widehat{u}) \in J_{2}(\widehat{V}),
$$

where $s, t \in \mathbb{R}, \widehat{u}=\left(x_{0}, u\right), \widehat{v}=\left(y_{0}, v\right) \in \widehat{V}=\mathbb{R} \oplus V$. The Jordan algebra $J_{2}(\widehat{V})$ is simple if $V \neq\{0\}$. Otherwise, $J_{2}(\widehat{V})=J_{2}(\{0\}) \cong \mathbb{R} \oplus \mathbb{R}$ is a sum of two simple algebras.

Proof. The isomorphism is given by

$$
\mathcal{H}_{2}(V) \ni X=\left(\begin{array}{cc}
t+x_{0} & v \\
v^{*} & t-x_{0}
\end{array}\right) \mapsto t 1+\left(x_{0}, v\right) \in J_{2}(\widehat{V}) .
$$

From the formula (12) it is straighforward to check the Jordan identity

$$
X\left(X^{2} Y\right)=X^{2}(X Y)
$$

for all $X, Y \in J_{2}(\widehat{V})$. Next assume that $J_{2}(\widehat{V})=A \oplus B$ is a direct sum of algebras. Then we can assume without loss of generality that $A$ contains an element of the form $X=1+\widehat{u}$. Using the formula (12), we see that $\left\{Y \in J_{2}(\widehat{V}) \mid X Y=0\right\} \supset B$ is non-zero if and only if $g(\widehat{u}, \widehat{u})=1$. In that case,

$$
\left\{Y \in J_{2}(\widehat{V}) \mid X Y=0\right\}=\mathbb{R}(1-\widehat{u})
$$

is one-dimensional. This shows that either $B=\{0\}$ or $A$ and $B$ are both onedimensional. The latter can only happen if $V=\{0\}$. 


\subsection{Rank 2 Vinberg cones}

The rank two Vinberg cone $\mathcal{V}_{2}(V)$ of the algebra $W=\mathcal{H}_{2}(V)$ is the (connected, but in general not convex) cone of formally positive definite matrices

$$
\mathcal{V}_{2}(V)=\left\{X \in W \mid x_{2}>0, \operatorname{det} X>0\right\} .
$$

In the Euclidean case, it coincides with

$$
\left\{X \in W \mid x_{1}>0, x_{2}>0, \operatorname{det} X>0\right\}=\left\{X=\left(t, x_{0}, v\right) \mid t>0, g(X, X)<0\right\},
$$

that is with the Lorentz cone of future directed timelike vectors of the Minkowski space $W$.

The set

$$
G=G_{2}(V)=\left\{A=\left(\begin{array}{cc}
a_{1} & a_{12} \\
0 & a_{2}
\end{array}\right) \in \mathcal{M}_{2} \mid a_{1}>0, a_{2}>0, a_{12} \in V\right\}
$$

of upper triangular matrices is a connected simply connected group. It acts in the vector space $\mathcal{M}_{2}$ by

$$
A: X \mapsto A \cdot X=A X A^{*}
$$

where

$$
A^{*}=\left(\begin{array}{cc}
a_{1} & 0 \\
a_{12}^{*} & a_{2}
\end{array}\right)
$$

is the conjugated lower triangular matrix. This action preserves the space $\mathcal{H}_{2}$ of Hermitian matrices. Note that $(A X) A^{*}=A\left(X A^{*}\right)$, which is no longer true in the rank 3 case, cf. Remark 2.

Lemma 3. The triangular group $G$ acts simply transitively on the cone $\mathcal{V}_{2}(V)$.

Proof. Note first that $(A B) C=A(B C)$ if $A$ and $B$ are lower triangular. By conjugation, the same is true if $B$ and $C$ are upper triangular. Furthermore, Proposition 2 implies that $(A B)^{*}=B^{*} A^{*}$ for all $A, B \in \mathcal{M}_{2}(V)$, using the natural inclusion $\mathcal{M}_{2}(V) \subset \mathcal{M}_{3}(V \oplus S)$ (e.g., with $S=0$ ). These properties easily imply that (13) is a group representation.

We have

$$
A \cdot \operatorname{Id}=A A^{*}=\left(\begin{array}{cc}
a_{1}^{2}+\left|a_{12}\right|^{2} & a_{2} a_{12} \\
a_{2} a_{12}^{*} & a_{2}^{2}
\end{array}\right)=\left(\begin{array}{cc}
x_{1} & v \\
v^{*} & x_{2}
\end{array}\right)=X \in \mathcal{V}_{2},
$$

and, for all $X \in \mathcal{V}_{2}$, the system of equations

$$
x_{1}=a_{1}^{2}+\left|a_{12}\right|^{2}, x_{2}=a_{2}^{2}, v=a_{2} a_{12}
$$

has unique solution

$$
a_{2}=\sqrt{x_{2}}>0, a_{12}=\frac{1}{\sqrt{x_{2}}} v, a_{1}=x_{2}^{-1}\left(x_{1} x_{2}-|v|^{2}\right)=x_{2}^{-1} \operatorname{det} X>0 .
$$




\subsection{The action of $\mathrm{Cl}(\widehat{V},-g)$ on a graded $\mathrm{Cl}(V)$-module}

Let $S=S_{0} \oplus S_{1}$ be a graded module over the (graded) Clifford algebra $\operatorname{Cl}(V)=$ $\mathrm{Cl}_{p, q}=\mathrm{Cl}(V)^{0}+\mathrm{Cl}(V)^{1}$.

We denote by

$$
\mu: V \otimes S \rightarrow S, \quad(v, s) \mapsto \mu_{v} s \in S
$$

the Clifford multiplication, which is odd. We consider elements $s=s_{0}+s_{1} \in S=$ $S_{0} \oplus S_{1}$ as column vectors $s=\left(s_{1}, s_{0}\right)^{t}$. Then we may define the product of a matrix $X \in W=\mathcal{H}_{2}(V)$ with a spin vector $s=\left(\begin{array}{l}s_{1} \\ s_{0}\end{array}\right)$ by

$$
\gamma_{X} s=X \cdot s=\left(\begin{array}{cc}
x_{1} & v \\
v^{*} & x_{2}
\end{array}\right)\left(\begin{array}{c}
s_{1} \\
s_{0}
\end{array}\right)=\left(\begin{array}{c}
x_{1} s_{1}+\mu_{v} s_{0} \\
-\mu_{v} s_{1}+x_{2} s_{0}
\end{array}\right) .
$$

Recall that the restriction of the metric $g(X, X)=-\operatorname{det} X$ to the traceless subspace $\mathcal{H}_{2}(V)^{0}=\widehat{V}=\mathbb{R} \oplus V$ has signature $(p+1, q)$.

Proposition 6. For $X \in \mathcal{H}_{2}(V)^{0}=\widehat{V}$, we have

$$
\gamma_{X}^{2}=g(X, X) \operatorname{Id} .
$$

Hence, $X \mapsto \gamma_{X}$ defines on $S$ the structure of a $\mathrm{Cl}(\widehat{V},-g)$-module.

Proof. Using the Clifford relation (1) and the product (14), we calculate

$$
X \cdot(X \cdot s)=\left(\begin{array}{c}
\left(x_{1}^{2}+|v|^{2}\right) s_{1}+\left(x_{1}+x_{2}\right) \mu_{v} s_{0} \\
\left(x_{2}^{2}+|v|^{2}\right) s_{0}-\left(x_{1}+x_{2}\right) \mu_{v} s_{1}
\end{array}\right)=\left(x_{0}^{2}+|v|^{2}\right) s,
$$

where we have used that $x_{1}=-x_{2}=x_{0}$.

Remark 3. Note that operators $\gamma_{X}, X \in \widehat{V}$, are symmetric with respect to any admissible scalar product $g_{S}$ on $S$ of type $\tau=-1$, as follows from the results of Section 2.2. Restricting the representation of $\mathrm{Cl}(\widehat{V},-g)$ on $S$ to the even subalgebra $\mathrm{Cl}(\widehat{V},-g)^{0}$, we can compare it to the representation of $\mathrm{Cl}(V)^{0}=\mathrm{Cl}\left(V, g_{V}\right)^{0}$ on $S$. The two representations are related by the canonical isomorphism $\mathrm{Cl}\left(V, g_{V}\right)^{0} \cong$ $\mathrm{Cl}\left(V,-g_{V}\right)^{0} \subset \mathrm{Cl}(\widehat{V},-g)^{0}$ induced by the map $v \mapsto i v$.

\subsection{The case when $V=\mathbb{K}$ is a division algebra and the Baez quantum- mechanical interpretation}

Following J. C. Baez and J. Huerta [6], [7] we consider the case when the Euclidean vector space $V=\mathbb{K} \in\{\mathbb{R}, \mathbb{C}, \mathbb{H}, \mathbb{O}\}$ is a division algebra. Then we may identify $\mathcal{H}_{2}(\mathbb{K})$ with the space of Hermitian forms in the right $\mathbb{K}$-module $\mathbb{K}^{2}$ and the Jordan multiplication $X \circ Y=\frac{1}{2}(X Y+Y X)$ defines on $\mathcal{H}_{2}(\mathbb{K})$ the structure of a Euclidean Jordan algebra with the Euclidean norm

$$
|X|=\sqrt{\langle X, X\rangle}=\sqrt{\operatorname{tr} X^{2}}=\left(x_{1}^{2}+x_{2}^{2}+2|v|^{2}\right) .
$$

John Baez gave the following quantum-mechanical interpretation of the cone $\mathcal{V}_{2}(\mathbb{K})$ $\subset \mathcal{H}_{2}(\mathbb{K})$. 
The irreducible graded $\mathrm{Cl}(\mathbb{K})$-module $S=S_{0} \oplus S_{1}$ (considered as a real Euclidean vector space) is identified with $\mathbb{K}^{2}=\mathbb{K} \oplus \mathbb{K}$, where $\mathbb{K}^{2}$ is considered as the right module of the division algebra $\mathbb{K}$, cf. Table 3.4 below. Then the Jordan algebra $\mathcal{H}_{2}(\mathbb{K})$ is identified with the algebra of Hermitian operators in the right $\mathbb{K}$-module $S=\mathbb{K} \oplus \mathbb{K}$. Baez identified them with the algebra of quantum (mixed) states of the quantum system. The "positive" states $\rho$, that is states which belong to the cone $V_{2}(\mathbb{K})$ and normalized by the condition $\operatorname{tr} \rho=1$, are considered as positive (on $\mathcal{V}_{2}(\mathbb{K})$ ) linear functionals $\rho(X)=\langle\rho, X\rangle=\operatorname{tr} \rho X$ (since the cone is selfdual). Such functionals $\rho$ are identified with observables and the value $\langle\rho\rangle_{X}:=\rho(X)=\langle\rho, X\rangle$ is considered as an expectation value of the observable in the state $X$.

We have a natural generalization of this interpretation to any special Vinberg Hermitian algebra $\mathcal{H}_{3}(V, S)=\operatorname{Herm}(W), W=V \oplus S$, cf. Section 2.2 for the notation. According to Section 2.2, this algebra is identified with a space $\left\{M_{A} \mid\right.$ $A \in \operatorname{Herm}(W)\} \cong \operatorname{Herm}(W)$ of symmetric operators acting on $W$. This space is not closed under the Jordan multiplication $\frac{1}{2}\left(M_{A} M_{B}+M_{B} M_{A}\right)$ in the space of all $g_{W}$-symmetric endomorphisms, but it is closed under the Vinberg multiplication $\frac{1}{2}(A B+B A)$. Following Baez, we may consider elements of $\operatorname{Herm}(W)$ as states and elements of the dual Vinberg cone $\mathcal{V}_{3}(W)^{*} \subset \operatorname{Herm}(W)^{*}$, which are by definition the linear functionals positive on $W$, as quantum observables of the theory.

TABLE 1 . Division algebras $\mathbb{K} \cong \mathbb{R}^{n}, n=1,2,4,8$, the Clifford algebra $\mathrm{Cl}(\mathbb{K})=\mathrm{Cl}_{n, 0}$, even Clifford algebra $\mathrm{Cl}_{n, 0}^{0}$, graded Clifford module $S=S_{0} \oplus S_{1}=\mathbb{K}^{2}$ and its decomposition $\mathbb{K} \oplus \mathbb{K}$ as a module of $\mathrm{Cl}_{n, 0}^{0}$, the spin group $\operatorname{Spin}(n)$ and the number $n(1,-1)=\left.n(\sigma, \tau)\right|_{\sigma=1, \tau=-1}$ of independent admissible symmetric $(\sigma=+1)$ bilinear forms on $S=\mathbb{K}^{2}$ of type $\tau=-1$. Similar tables can be given for other dimensions and signatures of $V$.

\begin{tabular}{|c|c|c|c|c|c|c|}
\hline$n$ & $\mathbb{K}$ & $\mathrm{Cl}_{n, 0}$ & $\mathrm{Cl}_{n, 0}^{0}$ & $S=S_{0} \oplus S_{1}$ & $\operatorname{Spin}(n)$ & $n(1,-1)$ \\
\hline 1 & $\mathbb{R}$ & $\mathbb{C}$ & $\mathbb{R}$ & $\mathbb{C}=\mathbb{R} \oplus \mathbb{R}$ & $\{\mathrm{Id}\}$ & 1 \\
\hline 2 & $\mathbb{C}$ & $\mathbb{H}$ & $\mathbb{C}$ & $\mathbb{H}=\mathbb{C} \oplus \mathbb{C}$ & $\mathrm{U}(1)$ & 1 \\
\hline 4 & $\mathbb{H}$ & $\mathbb{H}(2)$ & $\mathbb{H}$ & $\mathbb{H}^{2}=\mathbb{H} \oplus \mathbb{H}$ & $\mathrm{SU}(2) \times \mathrm{SU}(2)$ & 1 \\
\hline 8 & $\mathbb{O}$ & $\mathbb{R}(16)$ & $\mathbb{R}(8)$ & $\mathbb{R}^{16}=\mathbb{R}^{8} \oplus \mathbb{R}^{8}$ & $\operatorname{Spin}(8)$ & 1 \\
\hline
\end{tabular}

\section{Rank 3 Vinberg algebras and cones}

\subsection{Special Vinberg (rank 3) matrix algebras and special Vinberg cones}

As in Section 2, let $\left(S, g_{S}\right)$ be a graded $\mathrm{Cl}(V)$-module with an even admissible metric of type $\tau=-1$. We denote by $\mathcal{M}_{3}=\mathcal{A}(V, S)$ the vector space of matrices

$$
X=\left(\begin{array}{lll}
X_{11} & X_{12} & X_{13} \\
X_{21} & X_{22} & X_{23} \\
X_{31} & X_{32} & X_{33}
\end{array}\right),
$$

where $X_{i i} \in \mathbb{R}, X_{12} \in V, X_{21} \in V^{*}, X_{13} \in S_{1}, X_{31} \in S_{1}^{*}, X_{23} \in S_{0}, X_{32} \in S_{0}^{*}$. We fix the involutions

$$
*: V \rightarrow V^{*}, v \mapsto v^{*}=g v \quad \text { and } \quad *: S \rightarrow S^{*}, s \rightarrow s^{*}=g_{S} s
$$


and denote by $\mathcal{H}_{3}=\operatorname{Herm}(W) \subset \mathcal{A}(V, S)$ the subspace of Hermitian matrices of the form

$$
X=\left(\begin{array}{ccc}
X_{11} & X_{12} & X_{13} \\
X_{12}^{*} & X_{22} & X_{23} \\
X_{13}^{*} & X_{23}^{*} & X_{33}
\end{array}\right)=\left(\begin{array}{ccc}
x_{1} & X_{3} & X_{2} \\
X_{3}^{*} & x_{2} & X_{1} \\
X_{2}^{*} & X_{1}^{*} & x_{3}
\end{array}\right),
$$

where $X_{3}=X_{12} \in V, X_{1}=X_{23} \in S_{0}, X_{2}=X_{13} \in S_{1}$ and $X_{i j}^{*}:=\left(X_{i j}\right)^{*}$ denotes the element conjugated to $X_{i j}$.

\subsection{The group $G(V, S)$ of upper triangular matrices and its action in the space of Hermitian matrices}

Denote by $G=G(V, S) \subset \mathcal{M}_{3}$ the set of upper triangular matrices of the form

$$
A=\left(\begin{array}{ccc}
a_{11} & a_{12} & a_{13} \\
0 & a_{22} & a_{23} \\
0 & 0 & a_{33}
\end{array}\right)=\left(\begin{array}{ccc}
\alpha_{1} & a_{12} & a_{13} \\
0 & \alpha_{2} & a_{23} \\
0 & 0 & \alpha_{3}
\end{array}\right)
$$

where $\alpha_{1}, \alpha_{2}, \alpha_{3}>0$. It is a connected simply connected solvable Lie group with respect to matrix multiplication.

The group $G$ acts on the space $\mathcal{M}_{3}$ of matrices by left-multiplication

$$
(A, X) \mapsto L_{A} X=A X, \quad(A X)_{i j}=\sum_{k} a_{i k} X_{k j}
$$

It also acts by right-multiplication with the conjugate matrix:

$$
(A, X) \mapsto R_{A^{*}} X=X A^{*}, \quad\left(X A^{*}\right)_{i j}:=\sum_{k} X_{i k} a_{j k}^{*}
$$

where $\left(A^{*}\right)_{i j}=a_{j i}^{*}$ is the conjugate lower triangular matrix. However $L_{A}$ does not commute with $R_{A^{*}}$ in general, as shown in Remark 2 . So we cannot simply define a representation by $L_{A} \circ R_{A^{*}}$ or by $R_{A^{*}} \circ L_{A}\left(\neq L_{A} \circ R_{A^{*}}\right.$ in general). Nevertheless $T_{A} X=A X+X A^{*}$ defines a representation of the Lie algebra $\mathfrak{g}=$ Lie $G$ on the space of Hermitian matrices, see Theorem 1. We consider the representation $(A, X) \mapsto A \cdot X$ of $G$ on Hermitian matrices which is generated by it.

Lemma 4. For all $A \in G$ we have $A \cdot \operatorname{Id}=A A^{*}$.

Proof. It suffices to write $A=\exp B, B \in \mathfrak{g}$, and to compute

$A \cdot \mathrm{Id}=(\exp B) \cdot \mathrm{Id}=\left(\exp T_{B}\right) \mathrm{Id}=(\exp B) \cdot\left(\exp B^{*}\right)=(\exp B) \cdot(\exp B)^{*}$.

The following theorem extends the work of Vinberg [30], who considered the positive definite case.

Theorem 2. The orbit $\mathcal{V}=G \cdot \operatorname{Id}=\left\{A A^{*}, A \in G\right\}$ of the identity matrix is a homogeneous cone with the simply transitive action of $G$. If $g_{V}$ and $g_{S}$ are Euclidean metrics, then the cone is convex.

Definition 3. The cone $\mathcal{V}$ is called the special Vinberg cone (or cone of positively defined matrices) associated to the Clifford module $\left(S, g_{S}\right)$. 
Proof. Following Vinberg, we associate to a matrix $X=\left(X_{i j}\right) \in \mathcal{M}_{3}$ the matrix $X^{(2)} \in \mathcal{M}_{2}$, given by

$$
X^{(2)}=\left(\begin{array}{ll}
X_{11}^{(2)} & X_{12}^{(2)} \\
X_{21}^{(2)} & X_{22}^{(2)}
\end{array}\right)=\left(\begin{array}{ll}
X_{33} X_{11}-X_{13} \cdot X_{31} & X_{33} X_{12}-X_{13} \cdot X_{32} \\
X_{33} X_{21}-X_{23} \cdot X_{31} & X_{33} X_{22}-X_{23} \cdot X_{32}
\end{array}\right)
$$

In particular, for an upper triangular matrix $A$,

$$
A^{(2)}=\alpha_{3}\left(\begin{array}{cc}
\alpha_{1} & a_{12} \\
0 & \alpha_{2}
\end{array}\right)
$$

Similarly for the lower triangular matrix $A^{*}$ conjugate to $A$, the matrix $\left(A^{*}\right)^{(2)}=$ $\left(A^{(2)}\right)^{*}=\alpha_{3}\left(\begin{array}{cc}\alpha_{1} & 0 \\ a_{12}^{*} & \alpha_{2}\end{array}\right)$. Following Vinberg, consider the real-valued homogeneous polynomials of degree 1,2 , and 4 , given by

$$
\begin{aligned}
p_{3}(X)= & X_{33}, \\
p_{2}(X)= & X_{22}^{(2)}=X_{33} X_{22}-\left|X_{23}\right|^{2}, \\
p_{1}(X)= & \operatorname{det} X^{(2)}=X_{11}^{(2)} X_{33}^{(2)}-\left|X_{12}^{(2)}\right|^{2} \\
= & \left(X_{33} X_{11}-\left|X_{13}\right|^{2}\right)\left(X_{33} X_{22}-\left|X_{23}\right|^{2}\right) \\
& -\left(X_{33} X_{12}-X_{13} X_{32}\right)\left(X_{33} X_{21}-X_{23} X_{31}\right) .
\end{aligned}
$$

Here $\left|X_{23}\right|^{2}=g_{S}\left(X_{23}, X_{23}\right),\left|X_{13}\right|^{2}=g_{S}\left(X_{13}, X_{13}\right)$, and $\left|X_{12}\right|^{2}=g_{V}\left(X_{12}, X_{12}\right)$.

Lemma 5. If $X=A A^{*} \in \mathcal{V}, A \in G$, then

$$
X^{(2)}=A^{(2)}\left(A^{(2)}\right)^{*}=\alpha_{3}^{2}\left(\begin{array}{cc}
\alpha_{1}^{2}+\left|a_{12}\right|^{2} & \alpha_{2} a_{12} \\
\alpha_{2} a_{12}^{*} & \alpha_{2}^{2}
\end{array}\right)
$$

and

$$
p_{3}(X)=\alpha_{3}^{2}, p_{2}(X)=\alpha_{2}^{2} \alpha_{3}^{2}, p_{1}(X)=\left(\operatorname{det} A^{(2)}\right)^{2}=\alpha_{1}^{2} \alpha_{2}^{2} \alpha_{3}^{4} .
$$

Proof. For $A$ given by (15), the Hermitian matrix $X=A A^{*}$ is given by

$$
X=A A^{*}=\left(\begin{array}{ccc}
\alpha_{1}^{2}+\left|a_{12}\right|^{2}+\left|a_{13}\right|^{2} & \alpha_{2} a_{12}+a_{13} a_{23}^{*} & \alpha_{3} a_{13} \\
\alpha_{2} a_{12}^{*}+a_{23} a_{13}^{*} & \alpha_{2}^{2}+\left|a_{23}\right|^{2} & \alpha_{3} a_{23} \\
\alpha_{3} a_{13}^{*} & \alpha_{3} a_{23}^{*} & \alpha_{3}^{2}
\end{array}\right),
$$

where $\alpha_{i}:=a_{i i} \in \mathbb{R}$. From this one can directly check the claimed formulas.

Using the lemma, one can easily check the following proposition, which implies Theorem 2.

Proposition 7. There is a natural 1-1 correspondence between elements $A \in G$ and the points $X \in \mathcal{V}$ of the Vinberg cone. More precisely, for $X=A A^{*} \in \mathcal{H}$, the following formulas hold

$$
\alpha_{3}^{2}=p_{3}(X), \quad \alpha_{2}^{2}=\frac{p_{2}(X)}{p_{3}(X)}, \quad \alpha_{1}^{2}=\frac{p_{1}(X)}{p_{2}(X) p_{3}(X)},
$$




$$
\begin{gathered}
a_{13}=\frac{X_{13}}{\sqrt{p_{3}(X)}}, a_{23}=\frac{X_{23}}{\sqrt{p_{3}(X)}}, \\
a_{12}=\frac{X_{12}^{(2)}}{\sqrt{p_{2}(X) p_{3}(X)}}=\frac{\sqrt{p_{3}(X)}}{\sqrt{p_{2}(X)}} X_{12}-\frac{1}{\sqrt{p_{2}(X) p_{3}(X)}} X_{13} \cdot X_{23}^{*} .
\end{gathered}
$$

\subsection{Vinberg-Koszul characteristic function and invariant metric in special Vinberg cones}

We may consider $\mathcal{H}=\mathcal{H}_{3}=\operatorname{Herm}(W)=\mathbb{R}^{3} \oplus W, W=V \oplus S$, as a pseudoEuclidean vector space with the metric given by the sum of the Euclidean metric on $\mathbb{R}^{3}$ and the metric $g_{W}=g_{V} \oplus g_{S}$ on $W$. As shown in Section 2.1, the group $G=G(V, S)=G(W)$ acts as a linear group on $\mathcal{H}$. Since the group $G$ is a product of the diagonal subgroup $D=\left\{\operatorname{diag}\left(\alpha_{1}, \alpha_{2}, \alpha_{3}\right)\right\}$ and a unipotent subgroup $G^{\prime}$, the determinant $\operatorname{det}_{\mathcal{H}} A$ of an element $A \in G$, considered as a linear transformation of the vector space $\mathcal{H}$ depends only on the diagonal part $\Lambda=\operatorname{diag}\left(\alpha_{1}, \alpha_{2}, \alpha_{3}\right)$ of $A$ which acts on a matrix $X$ by

$$
\Lambda \cdot X=\left(\begin{array}{ccc}
\alpha_{1}^{2} x_{1} & \alpha_{1} \alpha_{2} X_{3} & \alpha_{1} \alpha_{3} X_{2} \\
\alpha_{1} \alpha_{2} X_{3}^{*} & \alpha_{2}^{2} x_{2} & \alpha_{2} \alpha_{3} X_{1} \\
\alpha_{1} \alpha_{3} X_{2}^{*} & \alpha_{2} \alpha_{3} X_{1}^{*} & \alpha_{3}^{2} x_{3}
\end{array}\right)
$$

where we are using the notation $x_{i}:=X_{i i}, X_{1}:=X_{23}, X_{2}:=X_{13}$ and $X_{3}:=X_{12}$. Let $\operatorname{dim} V=n, \operatorname{dim} S_{0}=\operatorname{dim} S_{1}=N$. Then we get:

\section{Lemma 6.}

$$
\operatorname{det}_{\mathcal{H}} A=\alpha_{1}^{2+n+N} \alpha_{2}^{2+n+N} \alpha_{3}^{2+2 N} .
$$

Remark 4. Recall that in the case $n=0$, the dimensions $N_{0}=\operatorname{dim} S_{0}$ and $N_{1}=$ $\operatorname{dim} S_{1}$ do not necessarily coincide. In that case we obtain the equality $\operatorname{det}_{\mathcal{H}} A=$ $\alpha_{1}^{2+N_{1}} \alpha_{2}^{2+N_{0}} \alpha_{3}^{2+N_{0}+N_{1}}$. For simplicity, we assume $n>0$ in the following.

The Vinberg-Koszul characteristic function of the cone $\mathcal{V}$ is defined as

$$
\chi(X)=\left(\operatorname{det}_{\mathcal{H}} A\right)^{-1} \text { where } X=A A^{*} .
$$

The functions $\alpha_{1}^{2}, \alpha_{2}^{2}, \alpha_{3}^{2}$ can be written as

$$
\begin{aligned}
\alpha_{3}^{2} & =p_{3}(X)=x_{3}, \\
\alpha_{2}^{2} & =\frac{p_{2}(X)}{p_{3}(X)}=\frac{x_{2} x_{3}-\left|X_{1}\right|^{2}}{x_{3}}, \\
\alpha_{1}^{2} & =\frac{p_{1}(X)}{p_{2}(X) p_{3}(X)},
\end{aligned}
$$

where the polynomials $p_{i}$ were defined in Section 4.2 and we recall that

$$
p_{1}(X)=\left(x_{1} x_{3}-\left|X_{2}\right|^{2}\right)\left(x_{2} x_{3}-\left|X_{1}\right|^{2}\right)-\left(x_{3} X_{3}-X_{2} \cdot X_{1}^{*}\right)\left(x_{3} X_{3}^{*}-X_{1} \cdot X_{2}^{*}\right) .
$$

By opening the brackets, using the associativity axiom (see Proposition 3), we compute 


$$
\begin{aligned}
p_{1}(X)= & x_{3}\left(x_{1} x_{2} x_{3}-x_{1}\left|X_{1}\right|^{2}-x_{2}\left|X_{2}\right|^{2}-x_{3}\left|X_{3}\right|^{2}\right) \\
& +\left(\left|X_{1}\right|^{2} \cdot\left|X_{2}\right|^{2}-\left(X_{2} \cdot X_{1}^{*}\right) \cdot\left(X_{1} \cdot X_{2}^{*}\right)\right) \\
& +x_{3}\left[\left(X_{2} \cdot X_{1}^{*}\right) X_{3}^{*}+X_{3} \cdot\left(X_{1} \cdot X_{2}^{*}\right)\right] \\
= & x_{3}\left[x_{1} x_{2} x_{3}-x_{1}\left|X_{1}\right|^{2}-x_{2}\left|X_{2}\right|^{2}-x_{3}\left|X_{3}\right|^{2}\right. \\
& +\underbrace{\left(X_{2} \cdot X_{1}^{*}\right) X_{3}^{*}+X_{3} \cdot\left(X_{1} \cdot X_{2}^{*}\right)}_{=2\left(X_{2} \cdot X_{1}^{*}\right) \cdot X_{3}^{*}}]
\end{aligned}
$$

Definition 4. The cubic polynomial $h(X)=p_{1}(X) / p_{3}(X)=\left(\alpha_{1} \alpha_{2} \alpha_{3}\right)^{2}$ is called the cubic potential of the cone $\mathcal{V}$ and the hypersurface $\mathcal{V}_{1}=\{h(X)=1\} \subset \mathcal{V}$ is called the canonical (or determinant) hypersurface of the cone.

\section{Theorem 3.}

i) The Vinberg cone $\mathcal{V}$ is described in terms of the Vinberg polynomials by the inequalities $p_{i}(X)>0, i=1,2,3$, or, equivalently, in terms of the cubic potential $h(X)$ by the three inequalities

$$
\begin{aligned}
& h(x)=x_{1} x_{2} x_{3}-x_{1}\left|X_{1}\right|^{2}-x_{2}\left|X_{2}\right|^{2}-x_{3}\left|X_{3}\right|^{2}+2\left(X_{2} \cdot X_{1}^{*}\right) \cdot X_{3}^{*}>0, \\
& x_{2} x_{3}-\left|X_{1}\right|^{2}>0 \quad \text { and } \quad x_{3}>0 .
\end{aligned}
$$

ii) The connected component of the level set $\{X \in \operatorname{Herm}(W) \mid h(X)=1\}$ which contains the identity matrix coincides with the canonival hypersurface $\mathcal{V}_{1}=\{X \in$ $\mathcal{V} \mid h(X)=1\}$ of the cone and $\mathcal{v}=\mathbb{R}^{>0} \cdot \mathcal{V}_{1}$.

iii) The characteristic function is given by

$$
\chi^{-1}(X)=\alpha_{1}^{2+n+N} \alpha_{2}^{2+n+N} \alpha_{3}^{2+2 N}=h(X)^{1+(n+N) / 2} p_{3}(X)^{(N-n) / 2} .
$$

Proof. The assertion ii) holds, since $v$ is connected and the function $h$ is homogeneous. The fact that the inequalities listed in i) hold on $v$ follows from (18). To prove the opposite inclusion it suffices to check that the set defined by the inequalities is connected. Equivalently, it suffices to show that the hypersurface

$$
\mathcal{H}:=\left\{\left.X \in \operatorname{Herm}(W)\left|h(X)=1, x_{3}>0, x_{2} x_{3}-\right| X_{1}\right|^{2}>0\right\}
$$

is connected and thus coincides with $\mathcal{V}_{1}$. The connectedness follows from the fact that the hypersurface $\mathcal{H}$ can be parametrized as the graph of a function over the connected open subset of $\mathbb{R}^{2} \oplus W=\left\{\left(x_{2}, x_{3}, X_{1}, X_{2}, X_{3}\right)\right\}$ defined by the inequalities $x_{3}>0, x_{2} x_{3}-\left|X_{1}\right|^{2}>0$ :

$$
x_{1}=\frac{1+x_{2}\left|X_{2}\right|^{2}+x_{3}\left|X_{3}\right|^{3}-2\left(X_{2} \cdot X_{1}^{*}\right) \cdot X_{3}^{*}}{x_{2} x_{3}-\left|X_{1}\right|^{2}} .
$$

Finally, using Lemma 6 and Definition 4, we calculate

$$
\chi^{-1}(X)=\operatorname{det}_{\mathcal{H}} A=\left(\alpha_{1} \alpha_{2} \alpha_{3}\right)^{2+n+N} \alpha_{3}^{N-n}=h(X)^{(2+n+N) / 2} x_{3}^{(N-n) / 2} .
$$




\section{Proposition 8.}

i) The cone $\mathcal{V}$ is naturally a homogeneous pseudo-Riemannian G-manifold with the metric $g_{\mathcal{V}}:=-\frac{1}{3} \log \partial^{2} h$, where $\partial^{2} h$ denotes the Hessian of $h$.

ii) The restriction $g_{\mathcal{H}}$ of the metric $g_{\mathcal{V}}$ to the hypersurface $\mathcal{H}=\mathcal{V}_{1}=\{h=$ $1\} \subset \mathcal{V}$ defines on $\mathcal{H}$ the structure of a homogeneous pseudo-Riemannian $G_{1}$ manifold, where $G_{1}=\left\{\alpha_{1} \alpha_{2} \alpha_{3}=1\right\} \subset G$ is the subgroup acting by unimodular transformations on the vector space $\operatorname{Herm}(W)$. The metric $g_{\mathcal{H}}$ can be equivalently defined by restriction of $-\frac{1}{3} \partial h$ to $\mathcal{H}$ without taking the logarithm.

iii) If the scalar product $g_{W}=g_{V} \oplus g_{S}$ is positive definite, the metrics $g_{\mathcal{V}}$ and $g_{\mathcal{H}}$ are complete Riemannian metrics, the domain $\mathcal{V}$ is convex and the cubic hypersurface $\mathcal{H}$ is strictly convex.

Proof. i), iii) First we note that $g_{\mathcal{V}}$ is $G$-invariant, as it is invariant under any linear transformation of $\operatorname{Herm}(W)$ preserving the cone $v \subset \operatorname{Herm}(W)$. It is also nondegenerate, which follows from a simple calculation of the Hessian at the point $\left(x_{1}, x_{2}, x_{3}, X_{1}, X_{2}, X_{3}\right)=(1,1,1,0,0,0)$. The same calculation shows that the metric $g_{\mathcal{V}}$ is positive definite if $g_{W}$ is. This implies the strict convexity of $\mathcal{H}$ and the convexity of $\mathcal{V}$.

Part ii) follows easily from i), using the homogeneity of $h$.

Remark 5. It follows from our discussion that the homogeneous projective special real manifolds classified in $[29,14]$ are precisely the homogeneous manifolds of the form $\left(\mathcal{H}, g_{\mathcal{H}}\right)$ arising in the above manner from special Vinberg cones of rank 3 and Vinberg cones of rank 2. They are of interest in high energy theoretical physics as they are exactly the homogeneous scalar manifolds of supergravity coupled to vector multiplets in five space-time dimensions.

Remark 6 . Under the supergravity $r$-map the above homogeneous projective special real manifolds give rise to homogeneous projective special Kähler manifolds, which in turn give rise to homogeneous quaternionic Kähler manifolds (of negative scalar curvature) under the supergravity c-map, see [29]. The homogeneous quaternionic Kähler manifolds are discussed more in detail in the Remark 7 below.

The r-map and the c-map are constructions which originate in the dimensional reduction of supergravity theories from 5 to 4 and from 4 to 3 space-time dimensions $d$, respectively. The reduction of the space-time dimension is accompanied by an increase of dimension of the corresponding scalar manifold, which is projective special real $(d=5)$, projective special Kähler $(d=4)$ or quaternionic Kähler $(d=3)$.

The composition of the $r$ - and the $c$-map is known as the $q$-map. It is a construction which associates a quaternionic Kähler manifold with every projective special real manifold. It can be also applied to the indefinite homogeneous cubic hypersurfaces associated with the indefinite homogeneous cones included in Theorem 3, cf. Proposition 8. The corresponding homogeneous indefinite quaternionic Kähler manifolds include symmetric spaces as well as non-symmetric examples.

Remark 7. Let $V$ be a Euclidean vector space of dimension $k \geq 0$. By choosing a $\mathbb{Z}_{2}$-graded $\mathrm{Cl}(V)$-module $S=S_{0} \oplus S_{1}$, we obtain homogeneous projective special real manifolds as convex connected components of the level sets of the cubic 
polynomials described above. These correspond under the q-map to homogeneous quaternionic Kähler manifolds [29], namely to the Alekseevsky spaces of rank 4, which are the spaces $\mathcal{W}(p, q), \mathcal{V}(\ell, k)$ and $\mathcal{V}(p, q ; \ell)[1,13]$. More precisely, taking $k=0, S$ is determined by two numbers $p=\operatorname{dim} S_{0}$ and $q=\operatorname{dim} S_{1}$. The corresponding spaces are the $\mathcal{W}(p, q)$. For $k \neq \equiv 0(\bmod 8)$ there is a unique irreducible $\mathbb{Z}_{2}$-graded $\mathrm{Cl}(V)$-module and an arbitrary module is determined by its multiplicity $\ell$. The corresponding spaces are the $\mathcal{V}(\ell, k)$. Finally, for $k \equiv 0$ $(\bmod 8)$ there are two non-equivalent irreducible $\mathbb{Z}_{2}$-graded $\mathrm{Cl}(V)$-modules and an arbitrary module $S$ is determined by the multiplicities $p$ and $q$ of those modules as submodules of $S$. These yield the spaces $\mathcal{V}(p, q ; \ell)$.

Apart from the rank 4 Alekseevsky spaces there are Alekseevsky spaces of rank 1, 2, and 3. The rank 3 spaces (denoted $\mathcal{T}(p)$ in [13], cf. Example 1 below) can be also obtained from the $q$-map [29] as well as the rank 2 space $\mathrm{G}_{2(2)} / \mathrm{SO}(4)$. The latter corresponds to the case when the projective special real manifold is reduced to a point. The remaining Alekseevsky spaces are the quaternionic hyperbolic spaces and the Hermitian symmetric spaces $\mathrm{SU}(m, 2) / \mathrm{S}(\mathrm{U}(m) \times \mathrm{U}(2))$.

\subsection{The triangular Lie algebra $\mathfrak{g}(V, S)=$ Lie $G(V, S)$ and its subalgebras}

The results of this section will be used for the study of group actions on subcones in Section 5.

The Lie algebra $\mathfrak{g}(V, S)$ of the group $G(V, S)$ has the gradation

$$
\mathfrak{g}(V, S)=\mathfrak{g}^{0}+\mathfrak{g}^{1}+\mathfrak{g}^{2}+\mathfrak{g}^{3}=\left\{\operatorname{diag}\left(\alpha_{1}, \alpha_{2}, \alpha_{3}\right) \mid \alpha_{i} \in \mathbb{R}\right\}+V_{23}+V_{12}+V_{13},
$$

where $\mathfrak{g}^{0}$ is the diagonal subalgebra and $V_{i j}=\mathcal{A}_{i j}, \mathcal{A}=\mathcal{A}(V, S)$. The group $G(V, S)$ is a direct product $G(V, S)=\left(\mathbb{R}^{>0} \cdot \mathrm{Id}\right) \times G_{1}(V, S)$ of the center and the normal subgroup $G_{1}(V, S)=\{\operatorname{det} A=1\}$ which acts by unimodular transformations. The Lie algebra $\mathfrak{g}_{1}(V, S)$ of $G_{1}(V, S)$ is given by

$$
\mathfrak{g}_{1}(V, S)=\mathfrak{d}+\mathfrak{n}=\mathfrak{d}+V_{23}+V_{12}+V_{13}
$$

where $\mathfrak{d}=\mathfrak{g}^{0} \cap \mathfrak{g}_{1}(V, S)$ is the 2-dimensional Cartan subalgebra and $\mathfrak{n}$ is the nilradical.

We will say that a subalgebra $\mathfrak{l} \subset \mathfrak{g}_{1}(V, S)$ has $\operatorname{rank} r=0,1,2$ if the intersection $\mathfrak{d}_{\mathfrak{l}}=\mathfrak{d} \cap \mathfrak{l}$ has dimension $r$ and $\mathfrak{l}=\mathfrak{d}_{\mathfrak{l}}+\mathfrak{l} \cap \mathfrak{n}$. Next we describe subalgebras of $\mathfrak{g}_{1}(V, S)$ of rank 1 and 2 .

Let $U_{i j} \subset V_{i j}(i<j)$ and $\mathfrak{d}^{\prime} \subset \mathfrak{d}$ be subspaces such that $U_{12} \cdot U_{23} \subset U_{13}$. Then

$$
\mathfrak{l}=\mathfrak{g}\left(\mathfrak{d}^{\prime}, U_{12}, U_{23}, U_{13}\right):=\mathfrak{d}^{\prime}+U_{12}+U_{23}+U_{13}
$$

is a subalgebra of $\mathfrak{g}_{1}(V, S)$. We call the subalgebra $\mathfrak{l}$ a standard subalgebra of $\mathfrak{g}_{1}(V, S)$ of rank $r$ and the subalgebra $\mathfrak{k}:=\mathbb{R} \cdot \operatorname{Id} \oplus \mathfrak{l}$ a standard subalgebra of rank $r+1$ of the triangular Lie algebra $\mathfrak{g}(V, S)$. The corresponding subgroups $L \subset G_{1}(V, S)$ and $K \subset G(V, S)$ are called standard.

Proposition 9. Let $\mathfrak{k}=\mathbb{R} d+\mathfrak{n}^{\prime}$ be a rank 1 subalgebra of $\mathfrak{g}_{1}(V, S)$, where $d=$ $\operatorname{diag}(\lambda, \mu,-(\lambda+\mu))$. Then either it is a standard subalgebra or one of the subalgebras described below. 
i) Up to scaling, $d=\operatorname{diag}(1,0,-1)$. Then the adjoint diagonal action of $\operatorname{ad}_{d}$ on $\mathfrak{n}=V_{12}+V_{23}+V_{13}$ is given by $\operatorname{ad}_{d}=\operatorname{diag}(1,1,2)$ and the associated rank one subalgebra $\mathfrak{l}$ of $\mathfrak{g}_{1}$ has the form

$$
\mathfrak{l}=\mathbb{R} d+U_{12}^{\varphi}+U_{23}+U_{13}
$$

where $U_{i j} \subset V_{i j}, U_{12}^{\varphi}=\left\{u+\varphi(u) \mid u \in U_{12}\right\}$ and $\varphi: U_{12} \rightarrow V_{23}$ is a linear map. The condition that $\mathfrak{l}$ is a subalgebra reads as

$$
\left[U_{12}^{\varphi}, U_{12}^{\varphi}\right] \subset U_{13},\left[U_{12}, U_{23}\right]=U_{12} \cdot U_{23} \subset U_{13}
$$

ii) $d=\operatorname{diag}(2,-1,-1)$ and $\operatorname{ad}_{d}=\operatorname{diag}(3,0,3)$. Then the associated subalgebra has the form

$$
\mathfrak{l}=\mathbb{R} d+U_{12}^{\varphi}+U_{13}+U_{23}
$$

where $U_{i j} \subset V_{i j}$ such that $U_{12} \cdot U_{23} \subset U_{13}$ and $U_{12}^{\varphi}=\left\{u+\varphi(u) \mid u \in U_{12}\right\}$ is the subalgebra $U_{12}$ twisted by a linear map $\varphi: U_{12} \rightarrow V_{13}$.

iii) $d=\operatorname{diag}(1,1,-2)$ and $\operatorname{ad}_{d}=\operatorname{diag}(0,3,3)$.

Then

$$
\mathfrak{l}=\mathbb{R} d+U_{12}+U_{23}^{\varphi}+U_{13}, U_{i j} \subset V_{i j},
$$

where $U_{23}^{\varphi}$ is the subalgebra $U_{23}$ twisted by a linear map $\varphi: U_{23} \rightarrow V_{13}$ and $U_{12}$. $U_{23} \subset U_{13}$.

Proof. A non-standard subalgebra which contains the element $d=\operatorname{diag}(\lambda, \mu,-(\lambda+$ $\mu)$ ) exists only if two of the $\operatorname{ad}_{d}$ eigenvalues $\lambda-\mu, 2 \mu+\lambda, 2 \lambda+\mu$ of of the eigenspaces $V_{12}, V_{23}, V_{13}$ coincide. There are three such cases. Analyzing them, we prove the proposition.

Corollary 2. Any rank 2 subalgebra $\mathfrak{l} \subset \mathfrak{g}_{1}(V, S)$ is standard.

\section{Sections of special Vinberg cones}

\subsection{Sections of Vinberg cones and special geometries of small cohomogeneity}

The intersection $v_{\Pi}=\mathcal{V} \cap \Pi$ of a Vinberg cone $\mathcal{V}=\mathcal{V}(V, S)$ with a subspace $\Pi \subset \mathcal{H}=\operatorname{Herm}(W), W=V \oplus S$, which contains a point $X \in \mathcal{V}$ is an open cone in $\mathcal{H}$, convex if $\mathcal{V}$ is convex.

\section{Definition 5.}

i) The cone $v_{\Pi}=\nu \cap \Pi$ is called a (conical) section or shortly a subcone of the Vinberg cone $\mathcal{V}$. If $\Pi$ is a hyperplane it is called a codimension 1 section or codimension 1 subcone, respectively.

ii) We say that a subcone $\mathcal{V}_{\Pi}$ has rank $r \leq 3$ if it is invariant under an $r$ dimensional subgroup of the diagonal group $D=\left\{\operatorname{diag}\left(\alpha_{1}, \alpha_{2}, \alpha_{3}\right) \mid \alpha_{i}>0\right\}$.

We are interested in (open) subcones $v_{\Pi}$ of small cohomogeneity $k$, that is cones for which the group of (linear) automorphisms acts with cohomogeneity $k$. We recall that the cohomogeneity of a group of transformations is the minimal codimension of an orbit. 
Example 1. Let $V=\{0\}, S=S_{0} \oplus S_{1}=\mathbb{R}^{p} \oplus\{0\}$ and consider the corresponding homogeneous projective special real manifold defined as

$$
\mathcal{H}:=\left\{h=1, x_{2}>0, x_{3}>0\right\} \subset \mathbb{R}^{3} \oplus S=\mathbb{R}^{3+p},
$$

where $h=x_{2}\left(x_{1} x_{3}-|Y|^{2}\right), x_{i} \in \mathbb{R}, Y \in \mathbb{R}^{p}$. Note that $Y=X_{13}=X_{2}$ in the notation of Section 4.3. Next we consider a hyperplane $\Pi=\Pi_{\lambda} \subset \mathbb{R}^{3+p}$ defined by an equation $x_{2}=\lambda$, where $\lambda=\lambda\left(x_{1}, x_{3}, Y\right)$ is a non-zero linear function in the variables $x_{1}, x_{3}$ and $Y$. Then

$$
\mathcal{H}_{\lambda}:=\mathcal{H} \cap \Pi \subset \Pi
$$

is a complete projective special real manifold, which was considered in $[15$, Thm. $2]$. Since the map $\left(x_{1}, x_{3}, Y\right): \Pi \rightarrow \mathbb{R}^{2+p}$ is a linear chart for $\Pi$, we see that the quadratic form $x_{1} x_{3}-|Y|^{2}$ restricts to a scalar product of signature $(1,1+p)$ on $\Pi$. The cubic polynomial $\left.h\right|_{\Pi}=\lambda\left(x_{1} x_{3}-|Y|^{2}\right)$ is reducible with a light-like, spacelike or time-like factor $\lambda$. This corresponds respectively to the cases b), c), and d) of [15, Thm. 2], up to a linear transformation. The automorphism group of $\mathcal{H}_{\lambda}$, that is the group of linear transformations of $\Pi$ which preserves the hypersurface $\mathcal{H}_{\lambda} \subset \Pi$, acts transitively on $\mathcal{H}_{\lambda}$ when $\lambda$ is light-like and with cohomogeneity one otherwise. Under the $q$-map one obtains the rank three Alekseevsky spaces $\mathcal{T}(p)$ in the first case and two series of complete quaternionic Kähler manifolds on which a certain group of isometries acts with cohomogeneity one [15, Cor. 28]. If $\lambda$ is time-like, then it is shown in [15, Thm. 3] that the full isometry group of the resulting complete quaternionic Kähler manifolds has no open orbit and is thus also of cohomogeneity one.

We continue to consider the special Vinberg algebra of $3 \times 3$ matrices $\mathcal{A}=$ $\mathcal{M}_{3}(V, S)$ associated with a $\mathrm{Cl}(V)$-module $S$ and the Vinberg cone $\mathcal{V}(V, S) \subset$ $\operatorname{Herm}(W) \subset \mathcal{A}$ of (formally) positive definite Hermitian matrices, cf. Theorem 3. Any rank 3 subcone is defined by subspaces $U_{12} \subset V_{12}=V, U_{23} \subset V_{23}=S_{0}$ and $U_{13} \subset V_{13}=S_{1}$. We denote such a subcone by

$$
\mathcal{V}\left(U_{12}, U_{23}, U_{13}\right)=\left\{X \in \mathcal{V}(V, S) \mid X_{i j} \in U_{i j}, i<j\right\} .
$$

To simplify notation, we will not indicate the subspace $U_{i j}=V_{i j}$. For example, we denote by $\mathcal{V}\left(U_{12}\right)$ the subcone $\mathcal{V}\left(U_{12}, V_{23}, V_{13}\right)$. Any rank 3 subcone of codimension 1 has the form $\mathcal{V}\left(U_{i j}\right)$, where $U_{i j}$ is a hyperplane in $V_{i j},(i j)=(12)$, (23) or (13).

The following proposition describes homogeneous rank 3 subcones of a Vinberg special cone $\mathcal{V}=\mathcal{V}(V, S)$.

Proposition 10. Let $\mathcal{V}=\mathcal{V}(V, S)$ be a special Vinberg cone. A subcone $\mathcal{V}\left(U_{12}, U_{23}\right.$, $\left.U_{13}\right)$ is a homogeneous cone if $U_{12} \cdot U_{23} \subset U_{13}$ and $U_{13} \cdot U_{23} \subset U_{12}$, cf. equation (11). In particular, the subcones of the form $\mathcal{V}\left(U_{12}\right), \mathcal{V}\left(U_{23}\right)$ are homogeneous.

Proof. The inclusion $U_{12} \cdot U_{23} \subset U_{13}$ ensures that

$$
G\left(U_{12}, U_{23}, U_{13}\right)=\left\{A \in G(V, S) \mid a_{i j} \in U_{i j}, i<j\right\}
$$

is a (rank 3) subgroup of the triangular group. The inclusion $U_{13} \cdot U_{23} \subset U_{12}$ ensures that it acts simply transitively in $\mathcal{V}\left(U_{12}, U_{23}, U_{13}\right)$. 
This proposition reduces the description of the cohomogeneity of codimension 1 cones of rank 3 to the cones $\mathcal{V}\left(U_{13}\right)$, where $U_{13}$ is a hyperplane of the spinor space $V_{13}=S_{1}$.

We consider an example. Let $\left(V, g_{V}\right)$ be a pseudo-Euclidean space of neutral signature $(m, m)$. We assume that $m \equiv 0$ or $3(\bmod 4)$, such that $S$ admits an even admissible scalar product of type $\tau=-1$. We fix a bi-isotropic decomposition $V=U \oplus U^{*}$ and identify the (unique up to equivalence) irreducible $\mathrm{Cl}(V)$-module with $S=\Lambda U$. We choose the grading such that $S_{0}=\Lambda^{\text {odd }} U, S_{1}=\Lambda^{\text {ev }} U$ if $m$ is even and $S_{0}=\Lambda^{\text {ev }} U, S_{1}=\Lambda^{\text {odd }} U$ if $m$ is odd. We consider the orthogonal hyperplane

$$
\Pi=1^{\perp}=\mathbb{R}^{3} \oplus V \oplus \sum_{i=0}^{m-1} \Lambda^{i} U \subset \operatorname{Herm}(W)=\mathbb{R}^{3} \oplus V \oplus S=\mathbb{R}^{3} \oplus V \oplus \Lambda U
$$

of the pure spinor $1 \in \Lambda^{m} U$. Then $S_{0} \subset \Pi$ and $\Pi \cap S_{1}$ is a hyperplane in $S_{1}$. We can define rank 3 subgroups $G\left(U^{*}, S_{0}, \Pi \cap S_{1}\right)$ and $G\left(V, \sum_{i=0}^{m-2} \Lambda^{i} U, \Pi \cap S_{1}\right)$, since the condition $U_{12} \cdot U_{23} \subset U_{13}$ of Proposition 10 is satisfied in both cases. However, only the second group satisfies also the condition $U_{13} \cdot U_{23} \subset S_{1} \cdot S_{0} \subset V=U_{12}$. Therefore the cone $\mathcal{V}\left(V, \sum_{i=0}^{m-2} \Lambda^{i} U, \Pi \cap S_{1}\right)$ admits the simply transitive group $G\left(V, \sum_{i=0}^{m-2} \Lambda^{i} U, \Pi \cap S_{1}\right)$ of automorphisms. As a consequence, the latter group acts with cohomogeneity $\leq m$ on the codimension 1 subcone $\mathcal{V}(\Pi) \subset \mathcal{V}$.

Question: Is it true that the above codimension 1 subcones have minimal cohomogeneity among codimension 1 subcones of rank 3 ?

Proposition 11. Let $K \subset G(V, S)$ be the standard Lie group generated by a rank 3 standard Lie subalgebra $\mathfrak{k}=\mathfrak{d}+U_{12}+U_{23}+U_{13}$. Then the orbit $K \cdot \operatorname{Id}=\left\{A A^{*} \mid\right.$ $A \in K\}$ is a homogeneous subcone, that is a section $\mathcal{V}_{\Pi}=\Pi \cap \mathcal{V}(V, S)$ of the cone $\checkmark$ with transitive action of the group $K$.

Remark 8. In the setting of Proposition 11, let $\tilde{\Pi}$ be a subspace of $\mathcal{H}_{3}=\operatorname{Herm}(W)$ which contains $\Pi$ as a hyperplane, then $K$ acts with cohomogeneity one the subcone $\tilde{\Pi} \cap \mathcal{H}_{3} \subset \mathcal{V}$. We consider cohomogeneity one subcones obtained in this way as trivial.

Remark 9. Let $K \subset G(V, S)$ be the subgroup associated to a subalgebra $\mathfrak{k}=$ $\mathbb{R}$ Id $\oplus \mathfrak{l}$ where $\mathfrak{l}$ is a rank one subalgebra of $\mathfrak{g}_{1}(V, S)$ described in Proposition 9. Then the orbit $K \cdot$ Id is a cone, but in general not an open cone in the vector $\operatorname{space} \operatorname{span}(K \cdot \operatorname{Id})$.

\subsection{Sections of the symmetric cone of positive definite quaternionic Hermitian matrices}

As an example, we consider rank 3 sections $\mathcal{V}\left(U_{12}, U_{23}, U_{13}\right)$ of the selfdual cone $\mathcal{V}\left(\mathbb{H}, \mathbb{H}^{2}\right)$ of quaternionic rank 3 Hermitian matrices. Here $V=\mathbb{H}, S=\mathbb{H}^{2}$ and hence $V_{i j}=\mathbb{H}$ for all $i<j$ in the notation of the previous section. We denote by $G\left(U_{12}, U_{23}, U_{13}\right) \subset G=G(\mathbb{H}, \mathbb{H}, \mathbb{H})$ the subset defined by the condition that the matrix elements $a_{i j}(i<j)$ belong to the subspaces $U_{i j} \subset \mathbb{H}$. 


\section{Proposition 12.}

i) The subset $G\left(U_{12}, U_{23}, U_{13}\right) \subset G$ is a subgroup if and only if $U_{12} \cdot U_{23} \subset U_{13}$.

ii) The subgroup $G\left(\mathcal{V}\left(U_{12}, U_{23}, U_{13}\right)\right)$ of $G$ which preserves the subcone $\mathcal{V}\left(U_{12}, U_{23}, U_{13}\right) \subset \mathcal{V}$ acts (simply) transitively if and only if $U_{13} \cdot U_{23} \subset U_{12}$.

Proof. i) It suffices to check that $G\left(U_{12}, U_{23}, U_{13}\right) \subset G$ is closed under multiplication if and only if $U_{12} \cdot U_{23} \subset U_{13}$, cf. the proof of Proposition 10, where one of the two implications was already mentioned.

ii) Similarly, one checks that the group $G\left(U_{12}, U_{23}, U_{13}\right)$ preserves the cone $v\left(U_{12}, U_{23}, U_{13}\right)$ if and only if $U_{13} \cdot U_{23} \subset U_{12}$ (again one of the implications was already mentioned in Proposition 10). This follows from the calculation of $X=A A^{*}$ for $A \in G\left(U_{12}, U_{23}, U_{13}\right)$ :

$$
X_{12}=\alpha_{2} a_{12}+a_{13} a_{23}^{*}, \quad X_{23}=\alpha_{3} a_{23}, \quad X_{13}=\alpha_{3} a_{13}
$$

From these formulas we also see that, under this assumption, $G\left(U_{12}, U_{23}, U_{13}\right)=$ $G\left(\mathcal{V}\left(U_{12}, U_{23}, U_{13}\right)\right)$. This proves ii).

Note that up to conjugation, any hyperplane of $\mathbb{H}$ has the form

$$
\mathbb{H}_{\theta}=\operatorname{span}\{\cos \theta 1+\sin \theta k, i, j\} \text {. }
$$

In particular, $\mathbb{H}_{0}=\operatorname{span}\{1, j, k\}$ contains complex fields $\operatorname{span}\{1, \sin \varphi i+\cos \varphi j\}$. We set $\mathbb{H}_{0}^{\prime}=\operatorname{span}\{j, k\}$. Denote by $\mathbb{C}=\operatorname{span}\{1, i\}$. Then $\mathbb{H}_{0}^{\prime}$ is a $\mathbb{C}$-module and $\mathbb{H}_{0}^{\prime} \cdot \mathbb{H}_{0}^{\prime}=\mathbb{C}$. This implies:

\section{Proposition 13.}

i) The section $\mathcal{V}\left(\mathbb{C}, \mathbb{H}_{0}^{\prime}, \mathbb{H}_{0}^{\prime}\right)$ is a homogeneous subcone.

ii) The section $\mathcal{V}\left(\mathbb{C}, \mathbb{H}_{0}^{\prime}, \mathbb{H}_{0}\right)$ is a cohomogeneity one subcone.

\section{References}

[1] Д. В. Алексеевский, Классификация кватернионных пространств с транзитивной разрешимой группой движений, Изв. АН СССР. Сер. матем. 39 (1975), вып. 2, 315-362. Engl. transl.: D. V. Alekseevskii, Classification of quaternionic spaces with a transitive solvable group of motions, Math. USSR-Izv. 9 (1975), no. 2, 297-339.

[2] D. V. Alekseevsky, V. Cortés, Classification of $N$-(super)-extended Poincaré algebras and bilinear invariants of the spinor representation of $\operatorname{Spin}(p, q)$, Comm. Math. Phys. 183 (1997), no. 3, 477-510.

[3] D. V. Alekseevsky, V. Cortés, Geometric construction of the r-map: from affine special real to special Kähler manifolds, Commun. Math. Phys. 291 (2009), 579-590.

[4] Shin-ichi Amari, Information Geometry and its Applications, Applied Mathematical Sciences, Vol. 194, Springer, Tokyo, 2016.

[5] S. A. Andersson, G. G. Wojnar, Wishart distributions on homogeneous cones, J. Theor. Probab. 17 (2004), 781-818.

[6] J. C. Baez, Division algebras and quantum theory, Foundations of Physics 42 (2012), no. 7, 819-855. 
[7] J. C. Baez, J. Huerta, Division Algebras and Supersymmetry, Part I: Proc. Symposia Pure Math. 81 (2010), 65-80; Part II: Adv. Theor. Math. Phys. 15 (2011), no. $5,1373-1410$.

[8] F. Barbaresco, Geometric theory of heat from Souriau Lie groups to thermodynamics and Koszul Hessian geometry: Applications, Information Geometry for Exponential Families, Entropy 18 (2016), 1-72.

[9] F. Barbaresco, Jean-Louis Koszul and the elementary structures of information geometry in: Geometric Structures of Information, Signals Commun. Technol., Springer, Cham, 2019, pp. 333-392.

[10] S. Bellucci, S. Ferrara, M. Günaydin, A. Marrani, SAM lectures on extremal black holes in $d=4$ extended supergravity, Proceedings of the INFN-Laboratori Nazionali di Frascati, 2007, 1-30, arXiv:0905.3739v1 (2009).

[11] A. Ben-Tal, A. Nemirovski, Lectures on Modern Convex Optimization. Analysis, Algorithms, and Engineering Applications, MPS/SIAM Series on Optimization, Society for Industrial and Applied Mathematics (SIAM), Philadelphia, PA; Mathematical Programming Society (MPS), Philadelphia, PA, 2001.

[12] P. Cartier, Vinberg algebras and combinatorics, IHES/M/09/34, 2009.

[13] V. Cortés, Alekseevskian spaces, Differential Geom. Appl. 6 (1996), no. 2, 129-168.

[14] V. Cortés, Homogeneous special geometry, Transform. Groups 1 (1996), no. 4, 337373.

[15] V. Cortés, M. Dyckmanns, M. Jüngling, D. Lindemann, A class of cubic hypersurfaces and quaternionic Kähler manifolds of co-homogeneity one, Asian J. Math., accepted 13/03/2020, arXiv:1701.7882 (2017).

[16] С. Г. Гиндикин, Анализ в однородных областях, УМН 19 (1964), вып. 4(118), 3-92. Engl. transl.: S. G. Gindikin, Analysis in homogeneous domains, Russian Math. Surveys 19 (1964), 1-83.

[17] P. Graczyk, H. Ishi, B. Kolodziek, Wishart law and variance function on homogeneous cones, Probability and Math. Statistics 39 (2019), 21-24.

[18] Э. Б. Винберг, В. В. Горбацевич, А. Л. Онищик, Строение групп и алгебр Ли, Итоги науки и техн., Совр. пробл. матем., Фунд. направл., т. 41, Группь Ли и алгебры Ли-3, ВИНИТИ, М., 1990, стр. 5-257. Engl. transl.: V. L. Gorbatsevich, A. L. Onishchik, E. B. Vinberg, Structure of Lie groups and Lie algebras, in: Lie Groups and Lie Algebras III, Encyclopaedia of Mathematical Sciences, Vol. 41, Springer-Verlag, Berlin, 1994, pp. 1-248.

[19] M. Günaydin, G. Sierra, P. K. Townsend, The geometry of $N=2$ Maxwell-Einstein supergravity and Jordan algebras, Nuclear Phys. B242 (1984), no. 1, 244-268.

[20] M. Günaydin, G. Sierra, P. K. Townsend, Exceptional supergravity theories and the magic square, Phys. Lett. B 133 (1983), no. 1-2, 72-76.

[21] E. Hairer, C. Lubich, G. Wanner, Geometric Numerical Integration. Structure-preserving Algorithms for Ordinary Differential Equations, Springer Series in Computational Mathematics, Vol. 31, Springer-Verlag, Berlin, 2002.

[22] P. Jordan, Über die Multiplikation quantenmechanischer Größen I, Zeitschrift für Physik 80 (1933), 285-291.

[23] P. Jordan, J. von Neumann, E. Wigner, On an algebraic generalization of the quantum mechanical formalism, Ann. of Math. (2) 35 (1934), no. 1, 29-64. 
[24] J.-L. Koszul, Domaines bornés homogènes et orbites de groupes de transformations affines, Bull. Soc. Math. France 89 (1961), 515-533.

[25] G. Lopes Cardoso, T. Mohaupt, Special geometry, Hessian structures and applications, Physics Reports 855 (2020), 1-141.

[26] D. Manchon, A short survey on pre-Lie algebras, in: Noncommutative Geometry and Physics: Renormalisation, Motives, Index Theory, ESI Lect. Math. Phys., Eur. Math. Soc., Zürich, 2011, pp. 89-102.

[27] T. Mohaupt, From special geometry to black hole partition functions, Springer Proc. Phys. 134 (2010), 165-241.

[28] J. Schray, The general classical solution of the superparticle, Class. Quant. Grav. 13 (1996), 27-38.

[29] B. de Wit, A. Van Proeyen, Special geometry, cubic polynomials and homogeneous quaternionic spaces, Commun. Math. Phys. 149 (1992), 307-333.

[30] Э. Б. Винберг, Теория однородных выпуклых конусов, Труды ММО 12 (1963), 303-358. [E. B. Vinberg, The theory of homogeneous convex cones, Trudy of Moscow Math. Soc. 12 (1963), 303-358 (Russian)].

[31] Э. Б. Винберг, Однородные конусы, Докл. АН СССР 133 (1960), 9-12. Engl. transl.: È. B. Vinberg, Homogeneous cones, Soviet Math. Dokl. 1 (1960), 787-790.

Funding Information Open Access funding enabled and ogranized by Projeckt DEAL.

Open Access This article is licensed under a Creative Commons Attribution 4.0 International License, which permits use, sharing, adaptation, distribution and reproduction in any medium or format, as long as you give appropriate credit to the original author(s) and the source, provide a link to the Creative Commons licence, and indicate if changes were made. The images or other third party material in this article are included in the article's Creative Commons licence, unless indicated otherwise in a credit line to the material. If material is not included in the article s Creative Commons licence and your intended use is not permitted by statutory regulation or exceeds the permitted use, you will need to obtain permission directly from the copyright holder. To view a copy of this licence, visit http://creativecommons.org/ licenses/by/4.0/.

Publisher's Note Springer Nature remains neutral with regard to jurisdictional claims in published maps and institutional affiliations. 